\title{
NUMERICAL APPROXIMATIONS OF THE DYNAMICAL SYSTEM GENERATED BY BURGERS' EQUATION WITH NEUMANN-DIRICHLET BOUNDARY CONDITIONS
}

\author{
Edward J. Allen ${ }^{1}$, John A. Burns ${ }^{2}$ And David S. Gilliam ${ }^{3}$
}

\begin{abstract}
Using Burgers' equation with mixed Neumann-Dirichlet boundary conditions, we highlight a problem that can arise in the numerical approximation of nonlinear dynamical systems on computers with a finite precision floating point number system. We describe the dynamical system generated by Burgers' equation with mixed boundary conditions, summarize some of its properties and analyze the equilibrium states for finite dimensional dynamical systems that are generated by numerical approximations of this system. It is important to note that there are two fundamental differences between Burgers' equation with mixed Neumann-Dirichlet boundary conditions and Burgers' equation with both Dirichlet boundary conditions. First, Burgers' equation with homogenous mixed boundary conditions on a finite interval cannot be linearized by the Cole-Hopf transformation. Thus, on finite intervals Burgers' equation with a homogenous Neumann boundary condition is truly nonlinear. Second, the nonlinear term in Burgers' equation with a homogenous Neumann boundary condition is not conservative. This structure plays a key role in understanding the complex dynamics generated by Burgers' equation with a Neumann boundary condition and how this structure impacts numerical approximations. The key point is that, regardless of the particular numerical scheme, finite precision arithmetic will always lead to numerically generated equilibrium states that do not correspond to equilibrium states of the Burgers' equation. In this paper we establish the existence and stability properties of these numerical stationary solutions and employ a bifurcation analysis to provide a detailed mathematical explanation of why numerical schemes fail to capture the correct asymptotic dynamics. We extend the results in [E. Allen, J.A. Burns, D.S. Gilliam, J. Hill and V.I. Shubov, Math. Comput. Modelling 35 (2002) 1165-1195] and prove that the effect of finite precision arithmetic persists in generating a nonzero numerical false solution to the stationary Burgers' problem. Thus, we show that the results obtained in [E. Allen, J.A. Burns, D.S. Gilliam, J. Hill and V.I. Shubov, Math. Comput. Modelling 35 (2002) 1165-1195] are not dependent on a specific time marching scheme, but are generic to all convergent numerical approximations of Burgers' equation.
\end{abstract}

Mathematics Subject Classification. 37L05, 65P30, 35B32, 35B41, 65M99, 65P40, 34B15.

Received January 2, 2012.

Published online July 30, 2013.

\footnotetext{
Keywords and phrases. Nonlinear dynamical system, finite precision arithmetic, bifurcation, asymptotic behavior, numerical approximation, stability, nonlinear partial differential equation, boundary value problem.

1 Supported in part by the National Science Foundation grant NSF-DMS 0718302.

Department of Mathematics and Statistics, Texas Tech University, Lubbock, TX 79409, USA. edward.allen@ttu.edu

2 Supported by the Air Force Office of Scientific Research grants FA9550-07-1-0273 and FA9550-10-1-0201 and by the DOE contract DE-EE0004261 under subcontract \# 4345-VT-DOE-4261 from Penn State University., Interdisciplinary Center for Applied Mathematics, Virginia Tech, Blacksburg, VA 24061, USA. jaburns@vt.edu

3 Supported in part by Air Force Office of Scientific Research grant FA9550-12-1-0114.

Department of Mathematics and Statistics, Texas Tech University, Lubbock, TX 79409, USA. david.gilliam@ttu.edu
} 


\section{INTRODUCTION}

As noted in the classic 1982 paper by Fletcher [26], Burgers' equation is often used as a test case for numerical methods to illustrate accuracy and convergence of a particular scheme. This is still true today as illustrated by the recent references (see $[23,32,35,38,44,48]$ and the references therein). It is interesting to note that although Burgers' equation has served as a great test case for numerous numerical algorithms, almost all papers along this line use Burgers' equation with Dirichlet boundary conditions. Also, Burgers' equation provides an excellent model problem to test conceptual flow control methods (see $[4,8,9,11,12,17,20,42]$ ) and it was first noted in these studies that Burgers' equation on finite intervals with Neumann boundary conditions raised some interesting new numerical questions. Neuman boundary control for fluid flows has been considered by several researchers over the past twenty years [33] (also see [27,34]). As noted by Fursikov [27], Neumann boundary control is known to cause a "local singularity" of the state at the boundary location and hence can lead to theoretical and computational challenges. In 1993 Burns and Marrekchi (see $[13,42]$ ) were investigating a Neumann boundary control for Burgers' equation when they encountered some unexpected numerical problems. In particular, they observed that every "standard" numerical method (finite elements, finite differences, modal expansion, etc.) always produced false asymptotic results. In particular, they demonstrated that a theoretically convergent numerical scheme can generate numerical steady state solutions that do not correspond to steady state solutions of the boundary value problem. Moreover, these numerically generated equilibria can (and often do) produce large errors in time marching schemes. These observations sparked a number of papers studying the dynamics and long time behavior of the controlled Burgers' equation with various Neumann and Robin boundary conditions (see $[4,6,10,15,16,18,19,21,22,41,43]$ ).

In $[2,3]$ it was shown that finite precision arithmetic (i.e. computing on a finite precision machine) could cause large numerical errors even when using "convergent numerical schemes." It is important to note that the problem is caused by finite precision arithmetic and is not due to "super-sensitivity" considered in [31,40]. In this paper we extend the results in [2] and show directly that the effect of finite precision arithmetic persists in generating nonzero numerical false solutions to the stationary Burgers' problem. Thus, the results obtained in [2] are not dependent on a specific time marching scheme. The importance of this analysis is to show that extreme care is required when using numerical methods to simulate the long time behavior of nonlinear convectiondiffusion systems on a finite precision machine. The long time behavior of a nonlinear dynamical system is best described in terms of invariant sets such as equilibria, limit sets and attractors. To illustrate the role that finite precision arithmetic can play in the approximation of nonlinear convection-diffusion systems, we focus on a case where the Burgers' equation has a single equilibrium which is the global attractor for the dynamical system. We conduct a bifurcation analysis of this system to provide a detailed mathematical explanation for the existence and stability properties of the numerical stationary solution for the Burgers' problem. Numerical results are given to illustrate the ideas and some open questions are discussed.

\section{BuRGERS' EQUATION WITH MIXED BOUNDARY CONDITIONS}

We consider the viscous Burgers' equation on the fixed finite interval $[0,1]$ with Neumann boundary condition at $x=0$ and Dirichlet condition at $x=1$. Note that Burgers' equation with a single Neumann boundary condition cannot be linearized by the Cole-Hopf transformation (see [20,43]). In particular, the Cole-Hopf transformation applied to Burgers' equation on a finite interval with a nonhomogeneous Neumann boundary condition fails to produce a well-posed linear equation (see [43]). Thus, Burgers' equation with a Neumann boundary condition is fundamentally a nonlinear system. We focus on two problems for this nonlinear system. The first is the time dependent initial boundary value problem and the second is the corresponding time independent (steady state) boundary value problem.

The system is governed by the viscous Burgers' equation

$$
z_{t}(x, t)=\nu z_{x x}(x, t)-z(x, t) z_{x}(x, t), 0 \leq x \leq 1,
$$


with initial condition

$$
z(x, 0)=\varphi(x)
$$

and boundary conditions

$$
z_{x}(0, t)=0, \quad z(1, t)=0 .
$$

The corresponding steady state (equilibrium) problem is defined by

$$
\nu v_{x x}(x)-v(x) v_{x}(x)=0,0 \leq x \leq 1,
$$

with boundary conditions

$$
v_{x}(0)=0, \quad v(1)=0 .
$$

Remark 2.1. Before discussing the properties of the dynamical system generated by this system, we make a few comments concerning Burgers' equation with various boundary conditions.

1. It was shown in $[22,41]$ that for the case of Dirichlet boundary conditions at both ends, Burgers' equation generates a dissipative dynamical system on $L_{2}(a, b)$, the zero function is the unique equilibrium and is the global attractor for this dynamical system (also see [47]). In particular, all solutions to the system (2.1) $-(2.3)$ converge to zero as $t \longrightarrow+\infty$ in the $L^{2}(a, b)$ norm. In addition, for $t>0$ it follows that $z(\cdot, t) \in H^{1}(a, b)$ and $\|z(\cdot, t)\|_{H^{1}(0,1)} \longrightarrow 0$ as $t \longrightarrow+\infty$. A very simple proof of this result is given in [21] using the classical Hopf-Cole transformation.

2. In the case with Neumann boundary conditions at both ends, Burgers' equation still generates a dynamical system on $L^{2}(a, b)$ but the dynamical system is not dissipative (see Cor. 3.3 in [41]). Indeed, every constant function is an equilibrium solution so there cannot be a bounded global absorbing ball. Nevertheless, in the paper [22] (also see the book [51]) it is proven that for any initial condition the corresponding solution converges uniformly, for $x$ in $[0,1]$, to a constant function. The constant of course depends on the initial condition. Moreover, when the initial function is asymmetric, i.e. the initial data satisfies $\varphi(x)=-\varphi([b-$ $a]-x)$ a.e. on $[a, b]$ one can show that this constant must be zero (see $[2,14]$ ). The proof of existence of the globally defined dynamical system is significantly more complicated due to the Neumann boundary conditions. In particular one cannot use the Hopf-Cole transformation in this case.

3. Burgers' equation with mixed boundary conditions lies somewhere in between the situations described in the two cases above. Due to the Neumann boundary condition at $x=0$ one encounters the same technical difficulties that arise in item 2. Namely, one is not able to obtain a global absorbing ball. On the other hand, because of the Dirichlet condition at $x=1$, one can establish the following result for Burgers' equation with mixed boundary conditions.

We shall make use of the following result which follows as a special case of Theorem 2.4 in [51].

Lemma 2.2. Let $\varphi(\cdot) \in L^{2}(0,1)$. If $z(x, t)$ is a solution to Burgers' system (2.1)-(2.3), then

$$
\lim _{t \rightarrow+\infty}\left\{\sup _{0 \leq x \leq 1}|z(x, t)|\right\}=0 .
$$

The following results provide the existence and stability of a globally defined dynamical system which is generated by Burgers' equation with mixed boundary conditions in (2.1)-(2.3) above.

Theorem 2.3. Burgers' system (2.1)-(2.3) generates a nonlinear dynamical system $\left\{S_{t}, t \geq 0\right\}$ on $L^{2}(0,1)$ and the following results hold:

(a) For every initial data $\varphi(\cdot) \in L^{2}(0,1)$ and $t>0, z(x, t)=\left[S_{t}(\varphi(\cdot))\right](x)$ is a smooth classical solution of $(2.1)-(2.3)$. 
(b) This dynamical system is Lyapunov stable. In particular, there exists a positive continuous monotone increasing function $a(\xi), \xi \geq 0$ such that $a(0)=0$ and

$$
\left\|S_{t} \varphi(\cdot)\right\| \leq a(\|\varphi(\cdot)\|) \text { for all } t \geq 0 \text { and all } \varphi(\cdot) \in L^{2}(0,1) .
$$

(c) The only equilibrium for this system is $z=0$ which is globally asymptotically stable in $H^{1}(0,1)$. Moreover, for every initial function $\varphi(\cdot) \in L^{2}(0,1)$ we have

$$
\lim _{t \rightarrow+\infty}\left\{\sup _{0 \leq x \leq 1}|z(x, t)|\right\}=0 .
$$

Proof of Theorem 2.3. The proof of parts (a) and (b) of Theorem 2.3 can be obtained by mimicking, almost line for line, the proofs given in [20] where the authors consider Burgers' equation with Robin boundary conditions. The only part of Theorem 2.3 that cannot be found directly in [20] is the proof of part (c). A simple calculation provides the general solution to (2.4) as

$$
v(x)=\sqrt{2 c_{0}} \tanh \left(\frac{\sqrt{2 c_{0}}}{2 \nu}\left(c_{1}-x\right)\right),
$$

where $c_{0}$ and $c_{1}$ are arbitrary constants. Thus, equilibrium solutions of (2.1) (i.e., functions satisfying (2.4)-(2.5)) are given by the two parameter family of functions defined in (2.8) above.

In order that $v$ satisfy the Dirichlet condition at $x=1$ we need $c_{1}=1$. Consequently, we need only to consider functions $h(\cdot)$ defined by

$$
h(x)=\sqrt{2 c_{0}} \tanh \left(\frac{\sqrt{2 c_{0}}}{2 \nu}(1-x)\right) .
$$

The derivative of $h(\cdot)$ defined by $(2.9)$ is given by

$$
h_{x}(x)=-\nu^{-1} c_{0} \operatorname{sech}^{2}\left(\frac{\sqrt{2 c_{0}}}{2 \nu}(1-x)\right),
$$

which cannot vanish at $x=0$ (unless $c_{0}=0$ ). Thus, the only stationary solution to the Burgers' problem (2.4), i.e., satisfying homogeneous Neumann boundary condition at $x=0$ and Dirichlet condition at $x=1$ is the zero function.

The proof of asymptotic stability follows from an argument obtained by employing a maximum principle together with the uniform Gronwall inequality. For this result one can show that the solutions are bounded in $H^{1}(0,1)$ norm for all $t>t_{0}>0$. Applying (2.6) from Lemma 2.2 above completes the proof.

Example 2.4. Observe that Theorem 2.3 above implies that if one sets $\nu=1 / 50$ and provides the initial data $z(x, 0)=15.2\left(1-x^{6}\right)$, then the solution to $(2.1)-(2.3)$ converges to zero. However, applying a standard spatial discretization with Crank-Nicolson time stepping (see e.g., Sect. 5.2), what actually happens is that the (numerical) solution converges to the function shown in Figure 1. One goal of this paper is to provide a complete analysis of this problem and show that this behavior is generic for the Burgers' equation with a Neumann boundary condition. Moreover, this behavior is not due to sensitivity (see $[31,40,49]$ ) nor metastability (see $[1,7,24,25,28-30,45])$. It is a consequence of finite precision arithmetic and can be understood through a bifurcation analysis of a nonhomogeneous Neumann boundary condition.

Consider now $h(\cdot)$ defined by (2.9) with small $\nu$ and/or large $c_{0}$. Observe for example that for a fixed $\nu=\hat{\nu}$

$$
\hat{\nu}^{-1} c_{0} \operatorname{sech}^{2}\left(\frac{\sqrt{2 c_{0}}}{2 \hat{\nu}}\right) \longrightarrow 0,
$$




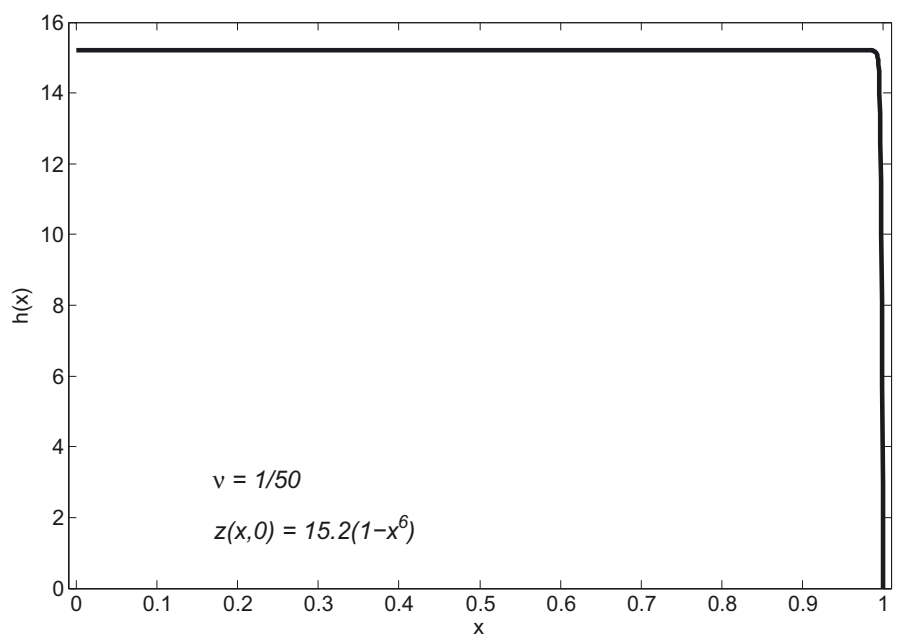

FIGURE 1. Nonzero asymptotic stationary solution.

as $c_{0} \longrightarrow+\infty$ and $c_{0} \longrightarrow 0$. Consequently, for each $\nu=\hat{\nu}$ and any sufficiently small positive number $\alpha$, there is a $c_{0}$ so that $h^{\prime}(\cdot)$ given by $(2.10)$ satisfies

$$
h^{\prime}(0)=-\hat{\nu}^{-1} c_{0} \operatorname{sech}^{2}\left(\frac{\sqrt{2 c_{0}}}{2 \hat{\nu}}\right)=-\alpha .
$$

Recall that $2^{-1022}$ is the smallest double precision floating point value that retains the full 53 bit accuracy. In the IEEE 754 standard [36], $2^{-1022}$ is also the smallest positive representable normal number in double precision. However, for example in MATLAB, positive floating point denormal numbers as small as $2^{-1074}$ are represented. We will use the notation RP to denote the smallest representable positive nonzero floating point number in the computational environment. For MATLAB, for example, $R P=2^{-1074}$.

The important observation is that on a computer with finite precision arithmetic, if $\alpha<\mathrm{RP}$, then $\alpha$ would be set equal to zero. In particular, for each $\nu=\hat{\nu}$ and any sufficiently small positive number $\alpha$, the nonzero function $h(x)$ given in (2.9) and satisfying (2.12) is a nonzero solution of the stationary Burgers' problem

$$
\begin{aligned}
& \nu v_{x x}(x)-v(x) v_{x}(x)=0, \\
& v_{x}(0)=-\alpha, \quad v(1)=0, \quad \alpha>0 .
\end{aligned}
$$

Thus, while the only stationary solution of time dependent Burgers' problem (2.1) with boundary condition (2.3) is the zero function, the time dependent system

$$
\begin{aligned}
& z_{t}(x, t)=\nu z_{x x}(x, t)-z(x, t) z_{x}(x, t), 0 \leq x \leq 1, \\
& z(x, 0)=\varphi(x) \\
& z_{x}(0, t)=-\alpha, \quad \alpha>0 \\
& z(1, t)=0,
\end{aligned}
$$

possesses nonzero stationary solutions defined by the system (2.13)-(2.14) and explicitly given by (2.9) where the constant $c_{0}$ is selected to satisfy (2.12).

We next turn to the relationship between the various parameters $\nu, c_{0}$ and $\alpha$. As it turns out there is an interesting bifurcation process that takes place that depends on these parameters. In order to keep the notation 
as simple as possible and to state the results in a concise form, we introduce two new parameters denoted by $\beta$ and $R$. Let $R$ be defined by

$$
R=R(\nu, V)=\frac{1}{\nu} \sqrt{\frac{c_{0}}{2}} \triangleq \frac{V}{\nu},
$$

where $V \triangleq \sqrt{\frac{c_{0}}{2}}$. It is helpful to think of $R$ as a "Reynolds number", $\nu$ as "dynamic viscosity" and $V$ as a "characteristic velocity", where characteristic distance and density are 1 . The parameter $\beta$ is defined by

$$
\beta=\sqrt{\nu^{-1} c_{0}}
$$

The significance of $\beta$ is that

$$
h^{\prime}(1)=-\beta^{2}
$$

which will be useful in the shooting method presented in Section 5.1. Note also that

$$
R=\sqrt{\frac{1}{2 \nu}} \beta
$$

Moreover, the condition that

$$
-\nu^{-1} c_{0} \operatorname{sech}^{2}\left(\frac{\sqrt{2 c_{0}}}{2 \hat{\nu}}\right)=-\alpha
$$

is the same as

$$
2 \nu R^{2} \operatorname{sech}^{2}(R)=\alpha,
$$

which implies that

$$
R \operatorname{sech}(R)=\sqrt{\alpha / 2 \nu} .
$$

Thus, $R$ depends implicitly on $\alpha$ and we shall make use of this dependency to conduct a bifurcation analysis of the system (2.15)-(2.18). The main significance of $R$ is that it simplifies the form of the stationary solution $h(\cdot)$ given in (2.9). This simplification aids in the presentation of the bifurcation analysis presented in Section 3 below.

Understanding the properties of the steady state problem (2.13) - (2.14) is essential to explaining the behavior of numerical approximations to Burgers' system (2.1)-(2.3) with a Neumann boundary condition. In the next section, it is shown that for $\alpha>0$ sufficiently small, there are two nonzero solutions to the steady state problem (2.13)-(2.14). One of these equilibrium solutions is "small", stable and converges to zero as $\alpha \longrightarrow 0$. The other equilibrium is "large", unstable and its norm approaches $+\infty$ as $\alpha \longrightarrow 0$.

\section{Bifurcation ANALYSiS}

Before addressing the bifurcation problem for the dynamical system (2.15) - (2.18), we provide a simple example to illustrate the type of bifurcation we expect to see for the Burgers' equation with "small" nonhomogeneous Neumann boundary condition (5.14).

\subsection{A simple motivating example}

The Logistic equation is a fundamental model in population dynamics and is useful in the present investigation as an example exhibiting a type of saddle node bifurcation that we observe for the Burgers problem (2.15) - (2.18). Let $p(t)$ denote the population of an organism at time $t$, with growth rate $r=1$ and carrying capacity $1 / K$. The Logistic equation is given by

$$
\frac{\mathrm{d} p(t)}{d t}=p(t)(1-K p(t))
$$




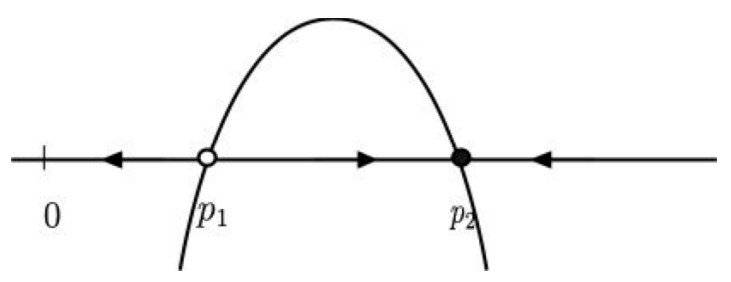

FIGURE 2. Equilibrium for the logistic equation with harvesting: $\alpha<\frac{1}{4 K}$.
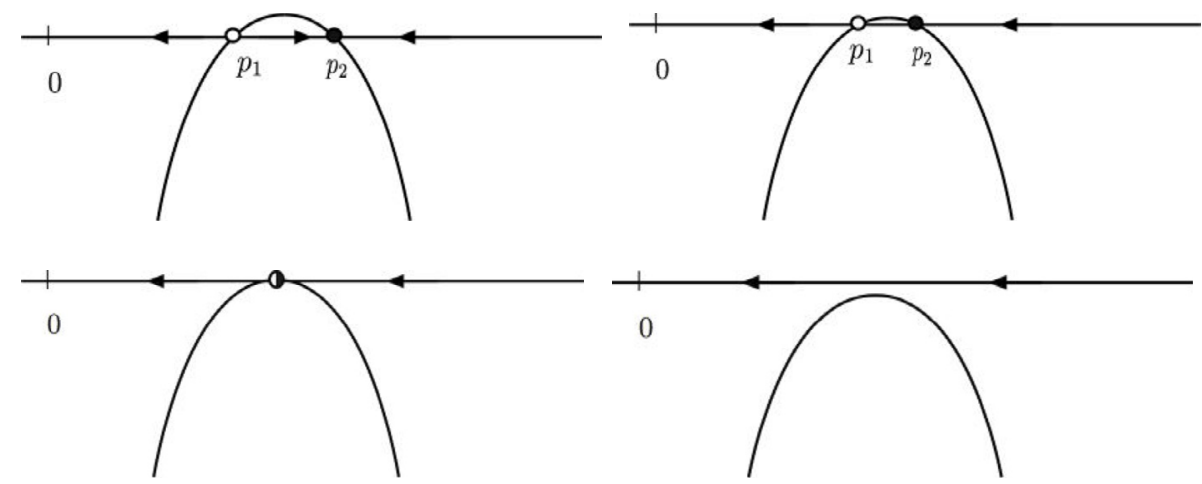

Figure 3. A simple bifurcation example.

and the dynamics of this equation are completely determined by the two equilibria at $p=0$ and $p=1 / K$. Namely, for any (positive) initial condition, as $t$ tends to infinity the population $p(t)$ tends to the carrying capacity $1 / K$.

On the other hand, the Logistic equation with a constant harvesting rate $\alpha \geq 0$ can be written as

$$
\frac{\mathrm{d} p(t)}{d t}=p(t)(1-K p(t))-\alpha
$$

and the equilibria are determined by $-K p^{2}+p-\alpha=0$, which gives

$$
p=\frac{1 \pm \sqrt{1-4 \alpha K}}{2 K} .
$$

Note that for $(1-4 \alpha K)>0$ there are two distinct equilibria, while for $(1-4 \alpha K)=0$ there is a single equilibrium and for $(1-4 \alpha K)<0$ all the equilibria disappear. For a fixed $K$ there are two equilibria when $\alpha<1 /(4 K)$ (i.e., when $(1-4 \alpha K)>0)$, only the zero equilibrium when $\alpha=1 /(4 K)$ and the equilibria disappear for $\alpha>1 /(4 K)$. Setting

$$
p_{1}=\frac{1-\sqrt{1-4 \alpha K}}{2 K}, \quad p_{2}=\frac{1+\sqrt{1-4 \alpha K}}{2 K},
$$

we can write $K p^{2}-p+\alpha=-K\left(p-p_{1}\right)\left(p-p_{2}\right)$ and the equilibrium for $0 \leq \alpha<1 /(4 K)$ are given in Figure 2 above.

Notice that as $\alpha$ tends to $1 /(4 K)$ the parabola decreases and the two equilibria coalesce to a single point at $\alpha=1 /(4 K)$ and then disappear altogether for $\alpha>1 /(4 K)$ (see Fig. 3).

As we shall show below, a similar bifurcation occurs for the dynamical system generated by Burgers' equation (2.15) - (2.18) with respect to the parameter $\alpha \geq 0$ that occurs in the nonhomogeneous Neumann boundary condition (5.14). However, unlike the previous example the two equilibrium "diverge" as $\alpha \longrightarrow 0$ and the 
small equilibrium converges to the stable zero equilibrium. Moreover, understanding this bifurcation is the key to understanding the role that finite precision computing plays in producing erroneous long term behavior of numerical approximations.

\subsection{Bifurcation analysis for Burgers' equation}

Recall that the parameter $R=R(\nu, V)$ is defined by

$$
R=\frac{1}{\nu} \sqrt{\frac{c_{0}}{2}} \triangleq \frac{V}{\nu}
$$

where $V \triangleq \sqrt{\frac{c_{0}}{2}}$. Thus, the stationary solution $h(\cdot)$ to $(2.13)-(2.14)$ is explicitly given by $(2.9)-(2.12)$ as

$$
h(x)=2 \nu R \tanh (R(1-x)) .
$$

Moreover,

$$
h^{\prime}(x)=-2 \nu R^{2} \operatorname{sech}^{2}(R(1-x))
$$

and since

$$
h^{\prime}(0)=-\alpha,
$$

it follows that

$$
2 \nu R^{2} \operatorname{sech}^{2}(R)=\alpha .
$$

If we take the square root of both sides we arrive at a simple formula that can be used to analyze the the bifurcation process. In particular, note that

$$
\sqrt{2 \nu} R \operatorname{sech}(R)=\sqrt{\alpha}
$$

and hence

$$
R \operatorname{sech}(R)=\sqrt{\alpha / 2 \nu} .
$$

Remark 3.1. To fully grasp the role that finite precision arithmetic plays in producing erroneous solutions to the Burgers' system (2.1)-(2.3), it is important to observe that in a floating point number system it is possible for a number $\alpha$ to be taken as zero while $\sqrt{\alpha}$ is not zero in the floating point system. In order to highlight this point later, for a fixed $\nu$ we define

$$
F(R)=F(R, \nu) \triangleq \sqrt{2 \nu} R \operatorname{sech}(R)
$$

and consider the equation

$$
F(R)=F(R, \nu) \triangleq \sqrt{2 \nu} R \operatorname{sech}(R)=\sqrt{\alpha} .
$$

Observe that for all $\nu$, the maximum of $F(R, \nu)$ occurs at the maximum of $f(R)=R \operatorname{sech}(R)$ and $f$ has a single critical point $R^{*}$ at the root of

$$
0=\frac{\mathrm{d} f}{d R}=\operatorname{sech}(R)(1-R \tanh (R))
$$

It follows that

$$
\max _{R} F(R, \nu)=\sqrt{2 \nu} \max _{R} f(R)=\sqrt{2 \nu} f\left(R^{*}\right) \quad \text { where } R^{*} \approx 1.1997 .
$$

The key point here is that although the maximum value $\max _{R} F(R, \nu)$ depends on $\nu$, the location of this maximum occurs when $R^{*} \approx 1.1997$. 

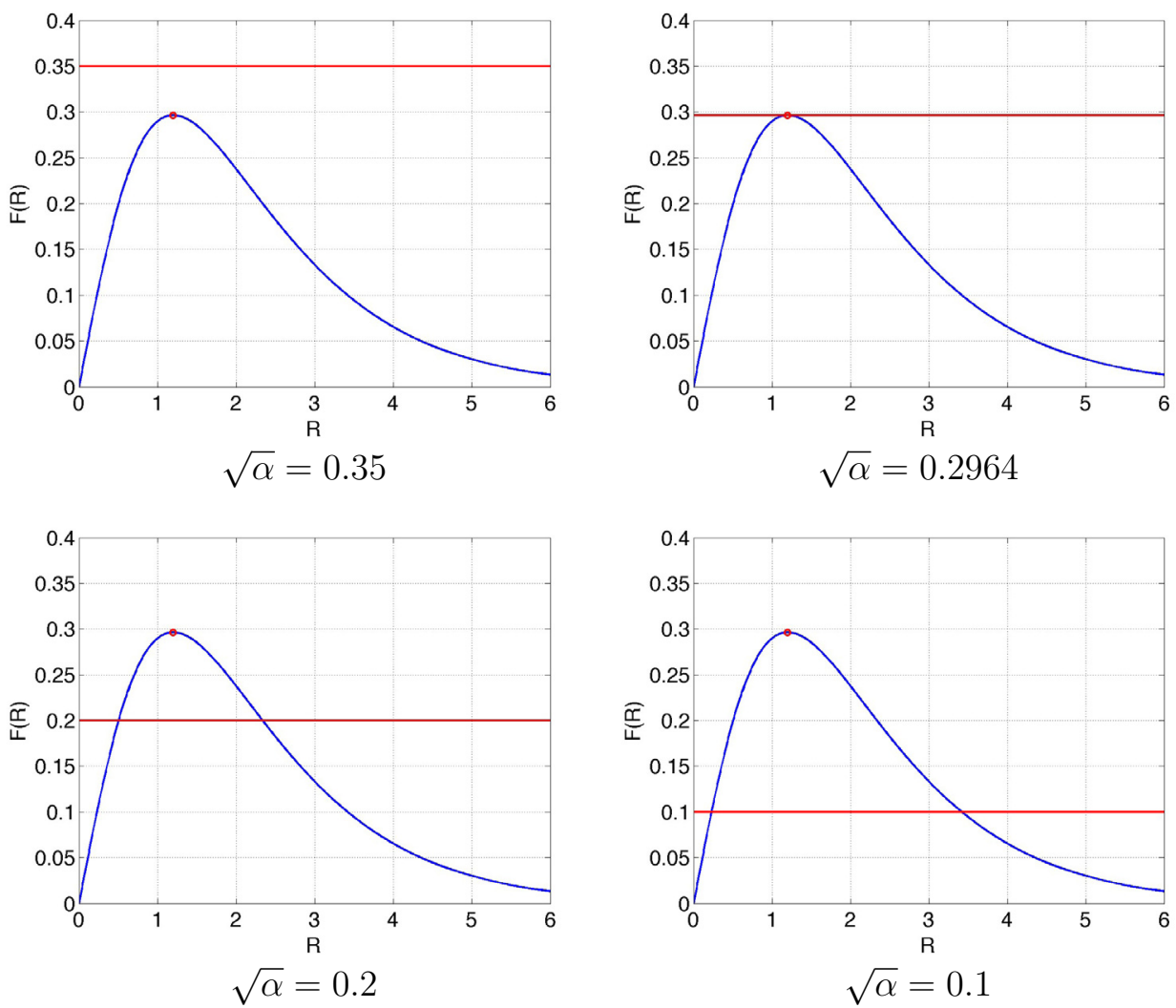

FiguRE 4. Bifurcation for burgers' equation in $\alpha$ for fixed $\nu$.

In Figure 4 we fix $\nu=1 / 10$ and note that the maximum of $F(R, \nu)$ occurs at $R^{*}$ with value $F(1.1997,1 / 10) \approx$ 0.2964. In Figure 4 we have plotted the graph of $F(R)$ along with the lines at heights corresponding to fixed values of $\sqrt{\alpha}$ given by $[0.35,0.2964,0.2,0.1]$. These plots demonstrate that the equation $F(R)=\sqrt{\alpha}$ provides a standard saddle node bifurcation in that for $\sqrt{\alpha}$ large there are no roots, for exactly one value of $\alpha$, corresponding to $R^{*}$ a single root appears and for all smaller values of $\alpha$ there are two roots, $R_{\mathcal{L}}$ and $R_{\mathcal{R}}$ with $R_{\mathcal{L}}<R_{\mathcal{R}}$. Furthermore, as $\alpha>0$ decreases to zero the two solutions $R_{\mathcal{L}}$ and $R_{\mathcal{R}}$ satisfy $R_{\mathcal{L}} \rightarrow 0$ and $R_{\mathcal{R}} \rightarrow \infty$, respectively.

Thus, for any fixed $\nu$ and for $\alpha>0$ small enough, equation (3.8) has two roots $R_{\mathcal{L}}$ and $R_{\mathcal{R}}$ and corresponding to these roots we obtain two (stationary) solutions to system (2.13)-(2.14) which are explicitly given by (2.9). In particular we have the two steady state solutions

$$
h_{\mathcal{L}}(x)=2 \nu R_{\mathcal{L}} \tanh \left(R_{\mathcal{L}}(1-x)\right)
$$

and

$$
h_{\mathcal{R}}(x)=2 \nu R_{\mathcal{R}} \tanh \left(R_{\mathcal{R}}(1-x)\right),
$$

respectively.

In Figures 5 and 6 below we provide bifurcation diagrams for this problem as a function of $\alpha$ for fixed $\nu=1 / 10$. In the next section we discuss the stability of these equilibrium and show that the upper branch corresponds to an unstable equilibrium while the lower branch is stable. 


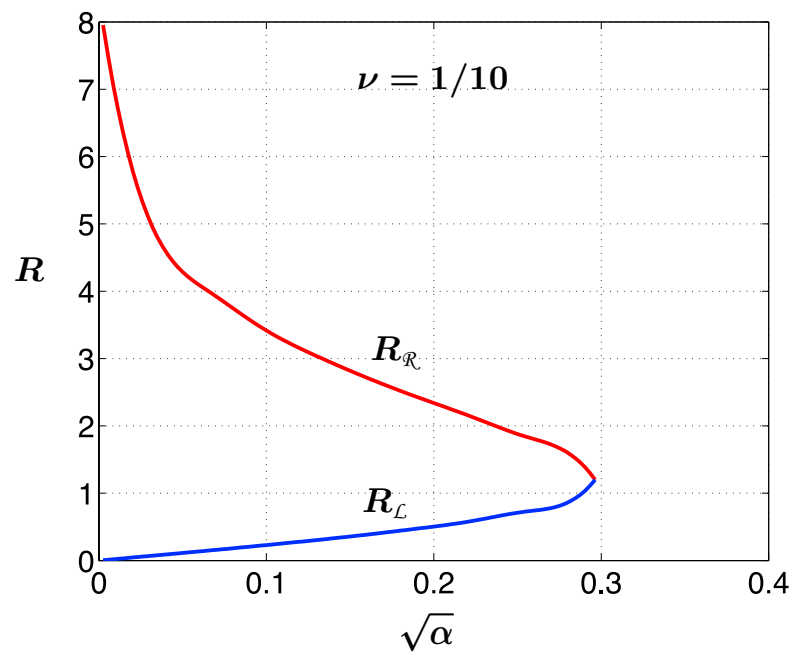

FiguRE 5. Bifurcation diagram in $R$.

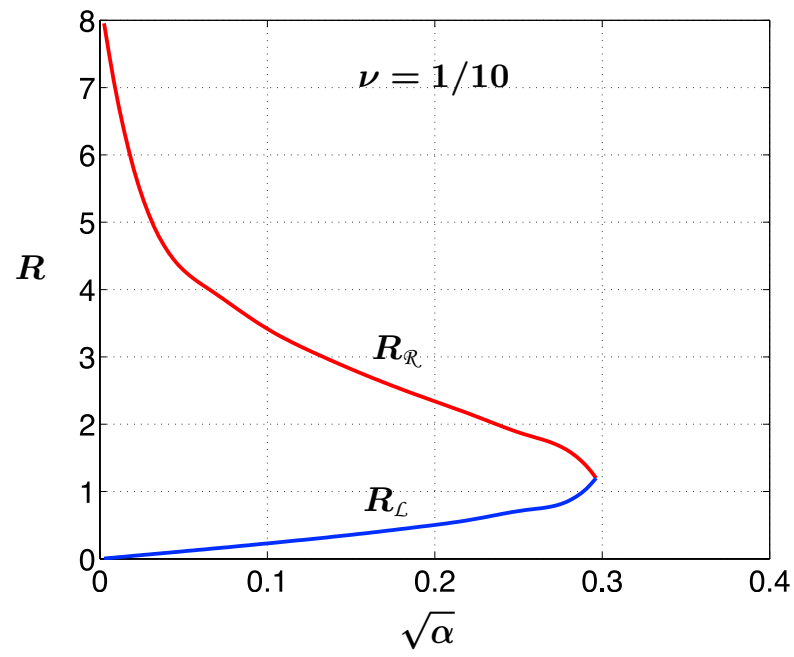

FiguRE 6. Bifurcation diagram in $\|h(\cdot)\|_{L^{2}(0,1)}$.

\section{Stability of THE EQUilibria}

In this section we consider the question of stability or instability of the equilibria $h_{\mathcal{L}}(\cdot)$ and $h_{\mathcal{R}}(\cdot)$ defined above. We show that the linearization about $h_{\mathcal{L}}(\cdot)$ has all negative eigenvalues so it is stable while the linearization about $h_{\mathcal{R}}(\cdot)$ has one positive eigenvalue and all other eigenvalues are negative eigenvalues.

For a given $R$, let $h(\cdot)$ be defined by (3.3). Set $z(x, t)=h(x)+\delta \zeta(x, t)$ and we find that, to first order in $\delta$, the function $\zeta$ satisfies

$$
\begin{aligned}
& \zeta_{t}(x, t)=\nu \zeta_{x x}(x, t)-(h \zeta)_{x}(x, t), \\
& \zeta_{x}(0, t)=0, \quad \zeta(1, t)=0 .
\end{aligned}
$$


Consider the linear differential operator

$$
L(\varphi(\cdot))=\nu \varphi_{x x}(\cdot)-(h(\cdot) \varphi(\cdot))_{x}
$$

and the associated eigenvalue problem

$$
\begin{aligned}
& L(\varphi(\cdot))=\nu \varphi_{x x}(\cdot)-(h(\cdot) \varphi(\cdot))_{x}=\lambda \varphi(\cdot), \\
& \varphi_{x}(0)=0, \quad \varphi(1)=0 .
\end{aligned}
$$

This problem is not in self-adjoint form and it is convenient to transform it into self-adjoint form. Applying the standard Liouville transformation (details are provided in the Appendix) we obtain the following equivalent self-adjoint eigenvalue problem:

$$
\begin{aligned}
& \psi_{x x}(x)-q(x) \psi(x)=\left(\nu^{-1} \lambda\right) \psi(x), \quad x \in[0,1], \\
& \psi_{x}(0)+k(R) \psi(0)=0, \quad \psi(1)=0,
\end{aligned}
$$

where $k(R)=R \tanh (R)$ and

$$
q(x)=\sqrt{2 \nu} R^{2}\left[1-2 \operatorname{sech}^{2}(R(1-x))\right] .
$$

Consider now the two cases where $R_{\mathcal{L}}<R^{*}$ and $R_{\mathcal{R}}>R^{*}$. Let $h=h_{\mathcal{L}}(\cdot)$ denote the steady state solution given by (3.11) when $R=R_{\mathcal{L}}$ and $h=h_{\mathcal{R}}(\cdot)$ denote the steady state solution given by (3.12) when $R=R_{\mathcal{R}}$. The following results establish linear stability of the lower branch of solutions defined by $h=h_{\mathcal{L}}(\cdot)$.

Remark 4.1. Since the eigenvalue problem (4.5)-(4.7) is symmetric we know that the eigenvalues are all real.

Theorem 4.2. For $h(\cdot)=h_{\mathcal{L}}(\cdot)$ with $R_{\mathcal{L}}$ sufficiently small, the eigenvalues of the Sturm-Liouville problem (4.5)-(4.6) are all negative. For $R=R^{*}$ the first (and largest) eigenvalue is zero and the rest are all negative.

Before we prove this result we recall some well known facts:

1. For a function $\varphi(\cdot) \in H^{1}(0,1)$ satisfying $\varphi(1)=0$ we have

(a) $\sup _{x \in[0,1]}|\varphi(x)| \leq\left\|\varphi^{\prime}(\cdot)\right\|$.

(b) $\|\varphi(\cdot)\| \leq\left\|\varphi^{\prime}(\cdot)\right\|\left(\right.$ where $\|\cdot\|$ denote the norm in $\left.L^{2}(0,1)\right)$.

2. The eigenvalues $\left\{\lambda_{j}\right\}_{j=1}^{\infty}$ of a regular Sturm-Liouville problem are simple (multiplicity one) and can be ordered in the form

$$
\lambda_{1}>\lambda_{2}>\ldots \lambda_{j} \rightarrow-\infty .
$$

3. The eigenfunction $\varphi_{1}(\cdot)$ associated with the first eigenvalue is not zero on the interval $[0,1)$.

Proof of Theorem 4.2. We show that, there exists an $R_{0}<R^{*}$ so that for all $R<R_{0}$ all eigenvalues of the Sturm-Liouville problem (4.5)-(4.6) are negative by showing that $\lambda_{1}<0$. Let $\psi_{1}(\cdot)$ denote the eigenfunction associated with $\lambda_{1}$. Hence

$$
\psi_{1}^{\prime \prime}(x)-q(x) \psi_{1}(x)=\nu^{-1} \lambda_{1} \psi_{1}(x) .
$$

Multiplying both sides of this equation by $\psi_{1}(x)$ and integrating we obtain

$$
\left\langle\psi_{1}^{\prime \prime}(\cdot), \psi_{1}(\cdot)\right\rangle-\left\langle q(\cdot) \psi_{1}(\cdot), \psi_{1}(\cdot)\right\rangle=\nu^{-1} \lambda_{1}\left\|\psi_{1}(\cdot)\right\|^{2},
$$

where $\langle\cdot, \cdot\rangle$ denotes the standard inner product on $L^{2}(0,1)$. Integrating by parts in the first term and applying the boundary conditions (4.6) at $x=0$ and $x=1$ yields

$$
\begin{aligned}
\nu^{-1} \lambda_{1}\left\|\psi_{1}(\cdot)\right\|^{2} & =-\left\|\psi_{1}^{\prime}(\cdot)\right\|^{2}-\left\langle q(\cdot) \psi_{1}(\cdot), \psi_{1}(\cdot)\right\rangle-\psi_{1}^{\prime}(0) \psi_{1}(0) \\
& =-\left\|\psi_{1}^{\prime}(\cdot)\right\|^{2}-\left\langle q(\cdot) \psi_{1}(\cdot), \psi_{1}(\cdot)\right\rangle+R \tanh (R)\left[\psi_{1}(0)\right]^{2} .
\end{aligned}
$$


Using the fact that $\left[\psi_{1}(0)\right]^{2} \leq\left\|\psi_{1}^{\prime}(\cdot)\right\|^{2}$, it follows that

$$
\nu^{-1} \lambda_{1}\left\|\psi_{1}(\cdot)\right\|^{2} \leq-\left\|\psi_{1}^{\prime}(\cdot)\right\|^{2}-\left\langle q(\cdot) \psi_{1}(\cdot), \psi_{1}(\cdot)\right\rangle+R \tanh (R)\left\|\psi_{1}^{\prime}(\cdot)\right\|^{2},
$$

or equivalently,

$$
\nu^{-1} \lambda_{1}\left\|\psi_{1}(\cdot)\right\|^{2} \leq-(1-R \tanh (R))\left\|\psi_{1}^{\prime}(\cdot)\right\|^{2}-\left\langle q(\cdot) \psi_{1}(\cdot), \psi_{1}(\cdot)\right\rangle .
$$

It is easy to show that

$$
(1-R \tanh (R))<1 \quad \text { for } 0<R<R^{*} .
$$

Indeed, $(1-R \tanh (R))$ is 1 at $R=0$ and monotone decreasing on $\left(0, R^{*}\right)$. Now we examine the term $-\left\langle q(\cdot) \psi_{1}(\cdot), \psi_{1}(\cdot)\right\rangle$. Note that we can assume without loss of generality that $\psi_{1}(x)>0$ for all $0 \leq x<1$ and that the function $q(\cdot)$ can be estimated. In order to investigate the function $q(x)=q(x, R)=$ $R^{2}\left[1-2 \operatorname{sech}^{2}(R(1-x))\right]$ we fix $R$. As $x$ varies from 0 to 1 the function $\operatorname{sech}^{2}(R(1-x))$ decreases monotonically from $\operatorname{sech}^{2}(R)$ to 1 . Therefore, for $R$ near zero $\operatorname{sech}^{2}(R)$ is near 1 and given $\epsilon>0$ there exists $R_{\epsilon}>0$ so that for all $R<R_{\epsilon}$ we have $(1-\epsilon)<\operatorname{sech}^{2}(R)<1$. Thus, if $R<R_{\epsilon}$ and $0 \leq x \leq 1$, then

$$
(1-\epsilon)<\operatorname{sech}^{2}(R(1-x))<1
$$

and

$$
-1<\left(1-2 \operatorname{sech}^{2}(R(1-x))<-1+2 \epsilon .\right.
$$

Consequently, we have that for all $0<R<R_{\epsilon}$ and $0 \leq x \leq 1$,

$$
R^{2}(1-2 \epsilon) \leq-q(x, R) \leq R^{2},
$$

which implies

$$
R^{2}(1-2 \epsilon)\left\|\psi_{1}(\cdot)\right\|^{2} \leq-\left\langle q(\cdot) \psi_{1}(\cdot), \psi_{1}(\cdot)\right\rangle \leq R^{2}\left\|\psi_{1}(\cdot)\right\|^{2} .
$$

In other words, for $R<R_{\epsilon}$ we have

$$
-\left\langle q(\cdot) \psi_{1}(\cdot), \psi_{1}(\cdot)\right\rangle \leq R^{2}\left\|\psi_{1}(\cdot)\right\|^{2} .
$$

Combining the estimate (4.12) with (4.8) and noting that

$$
0<(1-R \tanh (R))<1 \quad \text { for } 0<R<R^{*}
$$

we have

$$
\begin{aligned}
\nu^{-1} \lambda_{1}\left\|\psi_{1}(\cdot)\right\|^{2} & \leq-(1-R \tanh (R))\left\|\psi_{1}^{\prime}(\cdot)\right\|^{2}-\left\langle q(\cdot) \psi_{1}(\cdot), \psi_{1}(\cdot)\right\rangle \\
& \leq-(1-R \tanh (R))\left\|\psi_{1}^{\prime}(\cdot)\right\|^{2}+R^{2}\left\|\psi_{1}(\cdot)\right\|^{2} \\
& \leq-(1-R \tanh (R))\left\|\psi_{1}(\cdot)\right\|^{2}+R^{2}\left\|\psi_{1}(\cdot)\right\|^{2} \\
& =\left(-1+R \tanh (R)+R^{2}\right)\left\|\psi_{1}(\cdot)\right\|^{2} .
\end{aligned}
$$

Here on the third step we have used the fact that

$$
-(1-R \tanh (R))<0 \text { and }\left\|\psi_{1}(\cdot)\right\|^{2} \leq\left\|\psi_{1}^{\prime}(\cdot)\right\|^{2} .
$$

Thus we conclude that for $R<R_{\epsilon}$

$$
\lambda_{1} \leq \nu\left(-1+R \tanh (R)+R^{2}\right) .
$$

Finally, an elementary computation shows that $-1+R \tanh (R)+R^{2}<0$ for all $R<0.73485$. Consequently, if we select $R_{0}=\min \left\{R_{\epsilon}, 0.73485\right\}$ then for all $R<R_{0}$ we have $\lambda_{1}<0$ and this completes the proof. 


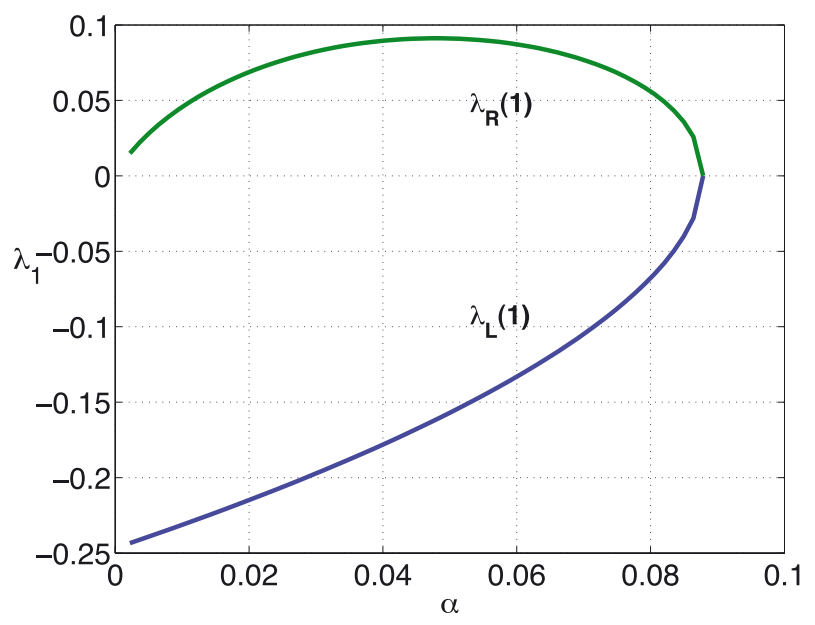

FiguRE 7. Bifurcation Diagram for $\nu=1 / 10$.

Consider the case where $R \geq R^{*}$. If $R=R^{*}>0$, then it follows from (3.9) that $R^{*} \tanh \left(R^{*}\right)=1$ and the Sturm-Liouville problem has zero as a simple eigenvalue with all other eigenvalues negative. To investigate the case where $R>R^{*}$, we set $\widetilde{\lambda}=\nu^{-1} \lambda$, so that the eigenvalue problem in $\widetilde{\lambda}$ is independent of $\nu$. Thus, we can easily approximate the eigenvalues $\tilde{\lambda}$ and the associated eigenfunctions for the Sturm-Liouville problem (4.5)-(4.6). The first five ( $\nu$ independent) eigenvalues $\tilde{\lambda}$ are listed in the following table:

$\begin{array}{cc}\tilde{\lambda}_{L} & \tilde{\lambda}_{R} \\ -2.1487 & 0.6878 \\ -21.8877 & -19.1508 \\ -61.3662 & -58.5841 \\ -120.5838 & -117.7991 \\ -199.5406 & -196.7556\end{array}$

Figure 7 provides a graphical display of the values of the first eigenvalue $\widetilde{\lambda}_{1}$ for both $R_{\mathcal{L}}$ and $R_{\mathcal{R}}$ for a sequence of values of $\alpha$. We denote these by $\widetilde{\lambda}_{R}(1)$ and $\widetilde{\lambda}_{L}(1)$, respectively. In Figure 7 we plotted the values of the eigenvalues for $\nu=1 / 10$ and for values of $\alpha$ between 0 and 0.1. This gives a graphical depiction of the bifurcation diagram. Notice that for other values of $\nu$ the eigenvalues given in Figure 7 are only scaled since $\lambda_{j}=\nu \widetilde{\lambda}_{j}$. Consequently, we have the following result.

Theorem 4.3. For $R=R^{*}$ the first (and largest) eigenvalue the Sturm-Liouville problem is zero and the rest are all negative. For $R>R^{*} \widetilde{\lambda}_{1}>0$ and all other eigenvalues are negative.

The analysis above provides a clear picture of the dynamical system generated by Burgers' equation with mixed boundary conditions (2.15) - (2.18) where the Neumann boundary condition (5.14) is parameterized by $\alpha \geq 0$. At $\alpha=0$ zero is the only equilibrium state, it is asymptotically stable and all solutions converge to zero. However, as soon as $\alpha$ becomes positive (say $\alpha=\mathrm{RP}$ ) two equilibria appear. The "small" state $h_{\mathcal{L}}(\cdot)$ is stable and the "large" state $h_{\mathcal{R}}(\cdot)$ is unstable. Moreover, the linearization about $h_{\mathcal{R}}(\cdot)$ has a one dimensional unstable manifold. Consequently, numerical methods that are designed to solve the problem with the zero Neumann boundary condition $\alpha=0$ when implemented on a finite precision machine where $\alpha=\mathrm{RP}$ are in fact approximating the more complex dynamical system (2.15)-(2.18). As we show in the next section, this property can, and often does, lead to erroneous numerical results regardless of the numerical algorithm used to simulate the system. 


\section{NUMERICAL SOLUTION}

We now consider the numerical solution of the stationary Burgers' problem (2.4) - (2.5) with homogeneous Neumann condition at $x=0$ and homogeneous Dirichlet condition at $x=1$. We know from the above analysis that the only solution to this problem is zero. The main objective of this section is to show that an accurate numerical procedure produces a sequence of iterates that can converge to a non-zero "numerical" solution which is actually a solution of the parameterized steady state system (2.13) - (2.14) with $\alpha$ sufficiently small. The point is that the iterative method provides a sequence of functions that converge to a function of the form (2.9) for which the numerical value of $\alpha$ in (2.12) is zero in the floating point number system of the computer. We begin with a shooting method for the problem (2.4)-(2.5).

\subsection{A shooting method}

In order to apply the shooting method it is more natural to reformulate the problem in terms of the following initial-value problem:

$$
\begin{aligned}
& \nu v_{x x}(x)-v(x) v_{x}(x)=0,0<x<1 \\
& v(1)=0, \quad v^{\prime}(1)=-\beta^{2}, \quad \beta>0
\end{aligned}
$$

where $-\beta^{2}$ is to be computed so that $v_{x}(0)=v_{x}(0 ; \beta)=0$. Specifically, $-\beta^{2}$ is an initial guess for the slope and (5.1) is solved from $x=1$ to $x=0$. After solving (5.1) from $x=1$ to $x=0$, the slope $-\beta^{2}$ is adjusted so that $G(\beta) \triangleq v_{x}(0 ; \beta)$ decreases to zero. Assuming that the initial value problem (5.1) is solved exactly, then

$$
v(x ; \beta)=\beta \sqrt{\frac{2}{\nu^{-1}}} \tanh \left(\beta \sqrt{\frac{\nu^{-1}}{2}}(1-x)\right)
$$

solves (5.1) with an initial guess of slope $-\beta^{2}$ and $v_{x}(x)=v_{x}(x, \beta)$ satisfies

$$
v_{x}(x ; \beta)=-\beta^{2} \operatorname{sech}^{2}\left(\beta \sqrt{\frac{\nu^{-1}}{2}}(1-x)\right)
$$

where $v_{x}(0 ; \beta)=-\beta^{2}$. Thus, we define $G(\beta) \triangleq v_{x}(0 ; \beta)$ so that

$$
G(\beta) \triangleq v_{x}(0 ; \beta)=-\beta^{2} \operatorname{sech}^{2}\left(\beta \sqrt{\nu^{-1} / 2}\right) .
$$

It follows that the shooting procedure, with exact initial-value solver, reduces to finding an algorithm to compute $\beta$ so that $G(\beta)=v_{x}(0 ; \beta)=-\beta^{2} \operatorname{sech}^{2}\left(\beta \sqrt{\nu^{-1} / 2}\right)=0$. Then, the solution to (5.1) is equal to $v\left(x ; \beta^{*}\right)$ where $\beta^{*}$ is a value such that $G\left(\beta^{*}\right)=0$. We now consider the problem of finding $\beta$ so that

$$
G(\beta)=-\beta^{2} \operatorname{sech}^{2}\left(\beta \sqrt{\nu^{-1} / 2}\right)=0 .
$$

Clearly the only solution to this equation is $\beta=0$. Nevertheless, we solve the problem $G(\beta)=0$ using Newton's method. Note that

$$
G(\beta)=-\beta^{2} \operatorname{sech}^{2}\left(\beta \sqrt{\nu^{-1} / 2}\right)
$$

implies

$$
G^{\prime}(\beta)=-\operatorname{sech}^{2}\left(\beta \sqrt{\nu^{-1} / 2}\right)\left(2 \beta-\left(\beta^{2} \sqrt{\nu^{-1} / 2}\right) \tanh \left(\beta \sqrt{\nu^{-1} / 2}\right)\right)
$$

and therefore Newton's method is defined by the iterations

$$
\beta_{j+1}=\beta_{j}-G^{\prime}\left(\beta_{j}\right)^{-1} G\left(\beta_{j}\right) .
$$


Let $\gamma\left(\beta_{j}\right)$ be defined by

$$
\gamma\left(\beta_{j}\right)=\left(\beta_{j} \sqrt{\nu^{-1} / 2}\right) \tanh \left(\beta_{j} \sqrt{\nu^{-1} / 2}\right)
$$

and simplifying (5.8) yields

$$
\beta_{j+1}=\beta_{j}\left(\frac{1 / 2-\gamma\left(\beta_{j}\right)}{1-\gamma\left(\beta_{j}\right)}\right) .
$$

On a digital computer we work within a finite floating point number system, $\mathcal{F}$. Let $\varnothing$ denote the base for a computer system and $t$ the number of digits. On the interval $\left[\varnothing^{m-1}, \varnothing^{m}\right]$, the floating point numbers are evenly spaced with separation $\varnothing^{m-t}$. In addition, for prescribed exponent limit $L$ for underflow, any number between $-\varnothing^{L-1}$ and $\varnothing^{L-1}$ is set equal to zero on the computer. For example, in Matlab, $\varnothing=2$ and $L=-1074$. Indeed, in the IEEE Standard 754, for denormalized floating point numbers with base $\varnothing=2, L=-149$ for single precision and $L=-1074$ for double precision.

Lemma 5.1. Fix $\nu>0$ and assume we are working in a floating point number system $\mathcal{F}$ with base $\varnothing$ and underflow exponent $L$ (so that any real number $|\alpha|<\varnothing^{L-1}$ is set equal to zero). If $\alpha^{*} \equiv \varnothing^{L-1}$, then there exists $\beta_{*}, \beta^{*}$ with $\beta_{*}<\beta^{*}$ and both solutions of

$$
\beta^{2} \operatorname{sech}^{2}\left(\beta \sqrt{\nu^{-1} / 2}\right)=\alpha^{*} .
$$

Moreover, $\left(\beta_{*} \operatorname{sech}\left(\beta_{*} \sqrt{\nu^{-1} / 2}\right)\right)^{2}$ and $\left(\beta^{*} \operatorname{sech}\left(\beta^{*} \sqrt{\nu^{-1} / 2}\right)\right)^{2}$ are both set equal to zero in the floating point system $\mathcal{F}$. In addition, for all $\beta<\beta_{*}$ and $\beta>\beta^{*}$ the expressions $\left(\beta \operatorname{sech}\left(\beta \sqrt{\nu^{-1} / 2}\right)\right)^{2}$ are likewise set equal to zero in $\mathcal{F}$.

Proof of Lemma 5.1. Denote by $\bar{\beta}$ the value of $\beta$ at which $G(\beta)$ achieves its maximum value. Notice that this is the value of $\beta$ at which $G^{\prime}(\beta)=0$ and using this formula a simple computation (see Fig. 8) shows that for every fixed $\nu$ there is a single critical point $\bar{\beta}$.

$$
G(\bar{\beta})=\max _{0 \leq \beta<\infty} G(\beta) .
$$

Observe that $\lim _{\beta \rightarrow 0} \gamma(\beta)=0$ and $\lim _{\beta \rightarrow \infty} \gamma(\beta)=\infty$ where $\gamma(\beta)$ is defined by (5.9) above and this completes the proof.

In view of the previous result, there are two cases to consider when analyzing the Newton iteration (5.8) and these are determined by whether the initial value $\beta_{0}$ satisfies $\beta_{0}<\bar{\beta}$ or $\beta_{0}>\bar{\beta}$. Thus, we have the following result.

Theorem 5.2. For $\beta_{0}$ sufficiently large or sufficiently small, the Newton iteration (5.8) in exact arithmetic provides a convergent numerical scheme. By that we mean that if $\beta_{0}$ is sufficiently large then $\beta_{j} \rightarrow \infty$ as $j \rightarrow \infty$ and if $\beta_{0}$ is sufficiently small, then $\beta_{j} \rightarrow 0$ as $j \rightarrow \infty$.

Proof of Theorem 5.2. We break the proof into two cases.

Case 1: $\boldsymbol{\beta}_{\mathbf{0}}>\overline{\boldsymbol{\beta}}$ Without loss of generality we can assume that $\beta_{0}$ is sufficiently large so that

$$
\gamma\left(\beta_{0}\right)=\left(\beta_{0} \sqrt{\nu^{-1} / 2}\right) \tanh \left(\beta_{0} \sqrt{\nu^{-1} / 2}\right)>1 .
$$

We want to show that the iterates in (5.8) diverge to infinity. Assume by way of contradiction that there exists a number $M$ so that $\beta_{j} \leq M$ for all $j$. Notice that under the above assumption $\gamma(\beta)>1$ for all $\beta \geq \beta_{0}$. Define the function

$$
g(\gamma)=\frac{1 / 2-\gamma}{1-\gamma}
$$




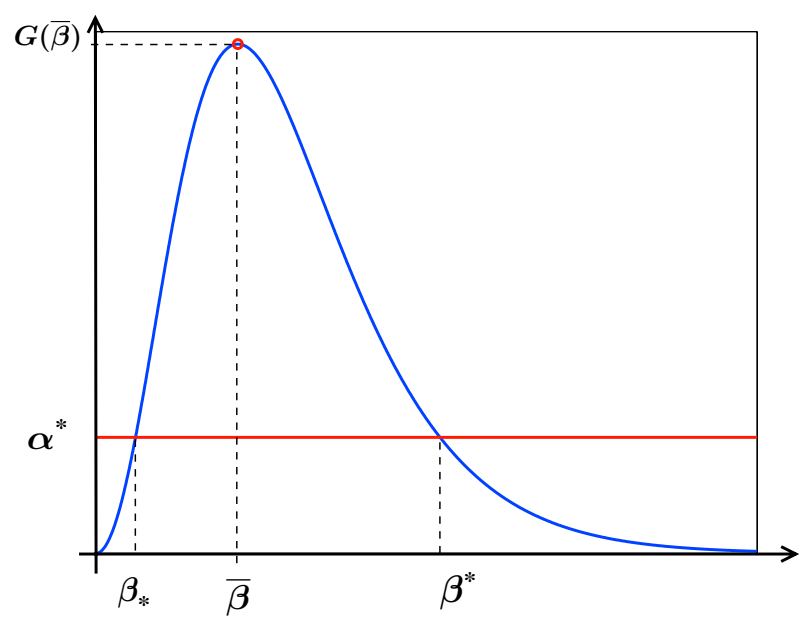

Figure 8. Bifurcation Plot in $\beta$.

and note that

$$
g^{\prime}(\gamma)=\frac{-1}{(1-\gamma)^{2}}<0 \text { for all } \gamma \neq 1
$$

Consequently, $g$ has a vertical asymptote at $\gamma=1$. An analysis of the graph of $g(\gamma)$ shows that it is always decreasing and for $1<\gamma<\infty$ we have $g(\gamma)>1$.

Recall from (5.8) that

$$
\beta_{j+1}=g\left(\gamma\left(\beta_{j}\right)\right) \beta_{j}, \quad j=0,1, \ldots
$$

and hence it follows that

$$
g\left(\gamma\left(\beta_{j}\right)\right) \geq g(\gamma(M)) \equiv(1+\delta)>1
$$

Thus,

$$
\begin{aligned}
\beta_{j+1} & =g\left(\gamma\left(\beta_{j}\right)\right) \beta_{j} \\
& \geq\left(\prod_{k=1}^{j} g\left(\gamma\left(\beta_{k}\right)\right)\right) \beta_{0} \\
& \geq g(\gamma(M))^{j} \beta_{0}=(1+\delta)^{j} \beta_{0} \stackrel{j \rightarrow \infty}{\longrightarrow} \infty
\end{aligned}
$$

which contradicts the assumption that the $\beta_{j}$ are bounded. Therefore, we must have that $\beta_{j} \rightarrow \infty$ as $j \rightarrow \infty$.

Case 2: $\boldsymbol{\beta}_{\mathbf{0}}<\overline{\boldsymbol{\beta}}$ Again, without loss of generality we assume that $\beta_{0}$ is small enough so that

$$
\gamma\left(\beta_{0}\right)=\left(\beta_{0} \sqrt{\nu^{-1} / 2}\right) \tanh \left(\beta_{0} \sqrt{\nu^{-1} / 2}\right)<\frac{1}{2}
$$

and it follows that for all $\beta \leq \beta_{0}$

$$
g(\gamma(\beta))<\frac{1}{2}
$$

To see why this holds, note that $\beta<\beta_{0}$ implies $\gamma(\beta)<\gamma\left(\beta_{0}\right)<1 / 2$ so that $\gamma(\beta)<1$ as well. Therefore, if $0<\gamma(\beta)<1$, then

$$
g(\gamma(\beta))=\frac{1 / 2-\gamma(\beta)}{1-\gamma(\beta)}<\frac{1}{2}
$$


and it follows that

$$
\frac{1}{2}-\gamma(\beta)<\frac{1}{2}(1-\gamma(\beta))
$$

Thus, we have

$$
g(\gamma)<\frac{1}{2} \text { for } \gamma<1
$$

and with the assumption on $\beta_{0}$ it follows that

$$
\beta_{j+1}=g\left(\gamma\left(\beta_{j}\right)\right) \beta_{j}<\left(\frac{1}{2}\right) \beta_{j}<\left(\frac{1}{2}\right)^{j} \beta_{0} \stackrel{j \rightarrow \infty}{\longrightarrow} 0
$$

and this completes the proof.

Remark 5.3. It is important to emphasize that the previous convergence results assumes (like all limiting analysis) one is using exact arithmetic. However, once the algorithm is run on a computer with a floating point number system $\mathcal{F}$ as described above, the iterations will stop as soon as

$$
G\left(\beta_{j}\right)<\alpha^{*}
$$

which is set equal to zero in $\mathcal{F}$ but it is not zero in exact arithmetic. This feature of computing on a computer with a floating point number system is exactly the reason for the erroneous numerical results produced by numerical algorithms for the Burger's system with Neumann boundary conditions. Although a (theoretically) convergent numerical algorithm is designed to solve the Burgers' system (2.1)-(2.3) with homogenous Neumann boundary condition $z_{x}(0, t)=0$, on a computer with a floating point number system the numerical method produces approximate solutions to the time dependent system

$$
\begin{aligned}
& z_{t}(x, t)=\nu z_{x x}(x, t)-z(x, t) z_{x}(x, t), 0 \leq x \leq 1, \\
& z(x, 0)=\varphi(x) \\
& z_{x}(0, t)=-\alpha, \quad \alpha \leq \alpha^{*}=\mathrm{RP}>0 \\
& z(1, t)=0 .
\end{aligned}
$$

This error is amplified for long time solutions and for the solution of the steady state system which should be zero.

Example 5.4. We use an iterative shooting scheme to solve the initial value problem (5.1),

$$
\begin{aligned}
& \nu v_{x x}(x)-v(x) v_{x}(x)=0, \quad 0<x<1 \\
& v(1)=0, \quad v^{\prime}(1)=-\beta^{2}, \quad \beta>0 .
\end{aligned}
$$

As an example we have set $\nu=1 / 500$ and in this example we will consider the Newton iterations for small and large values of the initial value $\beta_{0}$. The calculations were carried out using Matlab using both single and double precision. The simulations show that the iterations terminate at precisely the predicted values. As we have already mentioned, for Matlab the IEEE Standard 754, for denormalized floating point numbers with base $\varnothing=2$, has $L=-149$ for single precision and $L=-1074$ for double precision.

Note that for single precision we have $\varnothing^{L}=2^{-149}=1.4013 \times 10^{-45}$ and $\varnothing^{L-1}=2^{-149-1}=7.0065 \times 10^{-46}$ which is zero in single precision. Similarly for double precision we have $\varnothing^{L}=2^{-1074}=4.9407 \times 10^{-324}$ while $\varnothing^{L-1}=2^{-1074-1}=0$ in Matlab. The corresponding graph of $G(\beta)$ in this case is given in Figure 9.

In addition we have the following results for the Newton iteration. For single precision, we have the following values for the iterates in the case where $\beta_{0}=0.02$. 


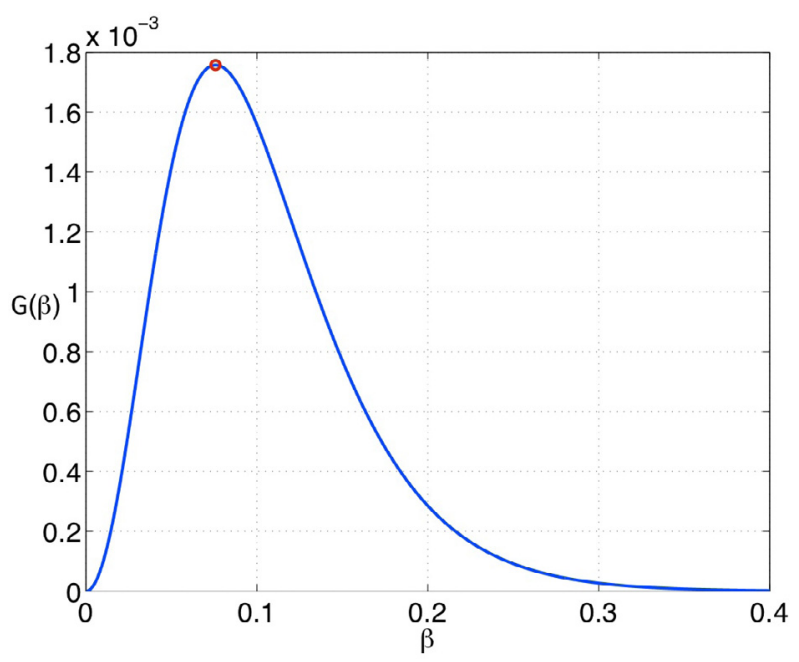

FiguRE 9. Graph of $G(\beta)$ for $\nu=1 / 500$.

\begin{tabular}{|c|c|c|}
\hline$j$ & $\beta_{j}$ & $G\left(\beta_{j}\right)$ \\
\hline 65 & $9.4242 \mathrm{e}-22$ & $8.8842 \mathrm{e}-43$ \\
\hline 66 & $4.7121 \mathrm{e}-22$ & $2.2141 \mathrm{e}-43$ \\
\hline 67 & $2.3560 \mathrm{e}-22$ & $5.6052 \mathrm{e}-44$ \\
\hline 68 & $1.1780 \mathrm{e}-22$ & $1.4013 \mathrm{e}-44$ \\
\hline 69 & $5.8901 \mathrm{e}-23$ & $2.8026 \mathrm{e}-45$ \\
\hline 70 & $2.9451 \mathrm{e}-23$ & $1.4013 \mathrm{e}-45$ \\
\hline 71 & $1.4725 \mathrm{e}-23$ & $0.0000 \mathrm{e}+00$ \\
\hline
\end{tabular}

If $\beta_{\mathcal{L}}=1.4725 \mathrm{e}-23$, then we obtain the corresponding numerical stationary solution $h_{\mathcal{L}}(x)$ depicted in Figure 10 .

Similarly, for $\beta_{0}=1$ we obtain the iterates

\begin{tabular}{|c|c|c|}
\hline$j$ & $\beta_{j}$ & $G\left(\beta_{j}\right)$ \\
\hline 67 & $3.1606 \mathrm{e}+00$ & $1.5667 \mathrm{e}-42$ \\
\hline 68 & $3.1929 \mathrm{e}+00$ & $5.7593 \mathrm{e}-43$ \\
\hline 69 & $3.2251 \mathrm{e}+00$ & $2.1160 \mathrm{e}-43$ \\
\hline 70 & $3.2574 \mathrm{e}+00$ & $7.8473 \mathrm{e}-44$ \\
\hline 71 & $3.2896 \mathrm{e}+00$ & $2.8026 \mathrm{e}-44$ \\
\hline 72 & $3.3219 \mathrm{e}+00$ & $1.1210 \mathrm{e}-44$ \\
\hline 73 & $3.3541 \mathrm{e}+00$ & $4.2039 \mathrm{e}-45$ \\
\hline 74 & $3.3863 \mathrm{e}+00$ & $1.4013 \mathrm{e}-45$ \\
\hline 75 & $3.4186 \mathrm{e}+00$ & $0.0000 \mathrm{e}+00$ \\
\hline
\end{tabular}




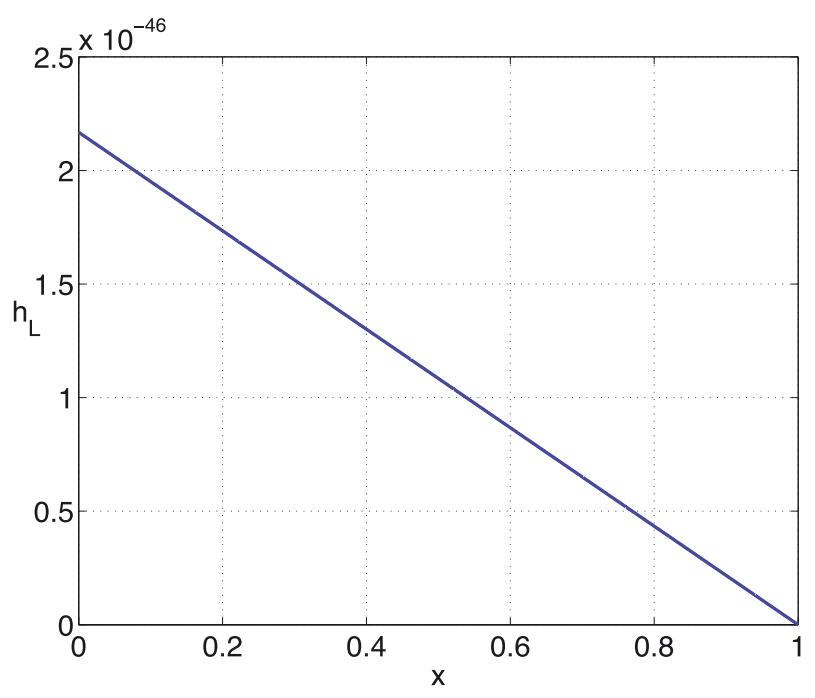

Figure 10. Plot of $h_{\mathcal{L}}(x)$.

If $\beta_{\mathcal{R}}=3.4186$, then we obtain the corresponding numerical stationary solution $h_{\mathcal{R}}(x)$ depicted in Figure 11 .

For the double precision case, if $\beta_{0}=0.02$, then we have the values for the iterates are given in the Table below.

\begin{tabular}{|c|c|c|}
\hline$j$ & $\beta_{j}$ & $G\left(\beta_{j}\right)$ \\
\hline 526 & $1.5828 \mathrm{e}-160$ & $2.5049 \mathrm{e}-320$ \\
\hline 527 & $7.9138 \mathrm{e}-161$ & $6.2648 \mathrm{e}-321$ \\
\hline 528 & $3.9569 \mathrm{e}-161$ & $1.5662 \mathrm{e}-321$ \\
\hline 529 & $1.9785 \mathrm{e}-161$ & $3.9031 \mathrm{e}-322$ \\
\hline 530 & $9.8923 \mathrm{e}-162$ & $9.8813 \mathrm{e}-323$ \\
\hline 531 & $4.9461 \mathrm{e}-162$ & $2.4703 \mathrm{e}-323$ \\
\hline 532 & $2.4731 \mathrm{e}-162$ & $4.9407 \mathrm{e}-324$ \\
\hline 533 & $1.2365 \mathrm{e}-162$ & $0.0000 \mathrm{e}+00$ \\
\hline
\end{tabular}

Also for the double precision case, if $\beta_{\mathcal{L}}=1.2365 \mathrm{e}-162$, we obtain the corresponding numerical stationary solution $h_{\mathcal{L}}(x)$ depicted in Figure 12. Observe that in this case we obtain a solution which appears to be identically zero (in double precision arithmetic).

Similarly, for $\beta_{0}=1$ we obtain the iterates 


\begin{tabular}{|c|c|c|}
\hline$j$ & $\beta_{j}$ & $G\left(\beta_{j}\right)$ \\
\hline 709 & $2.3590 \mathrm{e}+01$ & $2.3666 \mathrm{e}-321$ \\
\hline 710 & $2.3622 \mathrm{e}+01$ & $8.6956 \mathrm{e}-322$ \\
\hline 711 & $2.3653 \mathrm{e}+01$ & $3.2114 \mathrm{e}-322$ \\
\hline 712 & $2.3685 \mathrm{e}+01$ & $1.1858 \mathrm{e}-322$ \\
\hline 713 & $2.3717 \mathrm{e}+01$ & $4.4466 \mathrm{e}-323$ \\
\hline 714 & $2.3748 \mathrm{e}+01$ & $1.4822 \mathrm{e}-323$ \\
\hline 715 & $2.3780 \mathrm{e}+01$ & $4.9407 \mathrm{e}-324$ \\
\hline 716 & $2.3812 \mathrm{e}+01$ & $0.0000 \mathrm{e}+00$ \\
\hline
\end{tabular}

With a value of $\beta_{\mathcal{R}}=23.812$ we obtain the corresponding numerical stationary solution $h_{\mathcal{R}}(x)$ depicted in Figure 13.

This example clearly illustrates the role that a floating point number system has in producing erroneous solutions as predicted by Theorem 5.2 above. We turn now addressing erroneous long time solutions observed in numerous papers over the past twenty years for Burgers' equation with various Neumann and Robin boundary conditions (see $[2,3,6,10,13,14,16,19,21,22,41-43,46])$.

\subsection{Crank-Nicolson method}

As shown above, the time-dependent Burgers' problem (2.15) - (2.18) with nonhomogeneous Neumann boundary condition $z_{x}(0, t)=\alpha$ for sufficiently small $\alpha>0$ has two equilibrium solutions defined by the system (2.13)(2.14) and explicitly given by (2.9) where the constant $c_{0}$ is selected to satisfy (2.12). In particular, for $0<\alpha \ll 1$ these solutions have the form

$$
h(x)=2 \nu R \tanh (R(1-x))
$$

where $R=R(\nu, V)=\frac{1}{\nu} \sqrt{\frac{c_{0}}{2}}$.

On the other hand, for $\alpha=0$ the only equilibrium solution is the zero function and by Lemma 2.2 and Theorem 2.3 above, for any initial condition $\varphi(\cdot)$ solutions to $(2.1)-(2.3)$ must converge to zero as $t \longrightarrow+\infty$. However, it has been observed that most standard numerical methods developed for Burgers' equation fail to capture the correct long time behavior of this dynamical system (see $[2,3,6,10,13,14,16,19,21,22,41-43,46]$ ). Again, we show that this phenomenon is caused when theoretically convergent numerical schemes are implemented on a finite precision machine.

In particular, returning to problem $(2.1)-(2.3)$ we have

$$
\begin{aligned}
& z_{t}(x, t)=\nu z_{x x}(x, t)-z(x, t) z_{x}(x, t), 0 \leq x \leq 1, \\
& z(x, 0)=\varphi(x), \\
& z_{x}(0, t)=0, \\
& z(1, t)=0,
\end{aligned}
$$

and the corresponding time independent Burgers' problem is

$$
\begin{aligned}
& \nu z_{x x}(x)-z(x) z_{x}(x)=0 \\
& z_{x}(0)=0, \quad z(1)=0 .
\end{aligned}
$$

Recall that the only solution to this later problem is the zero function. One possible numerical method for solving the time independent Burgers' problem (5.21)-(5.22) would be to solve the time dependent 


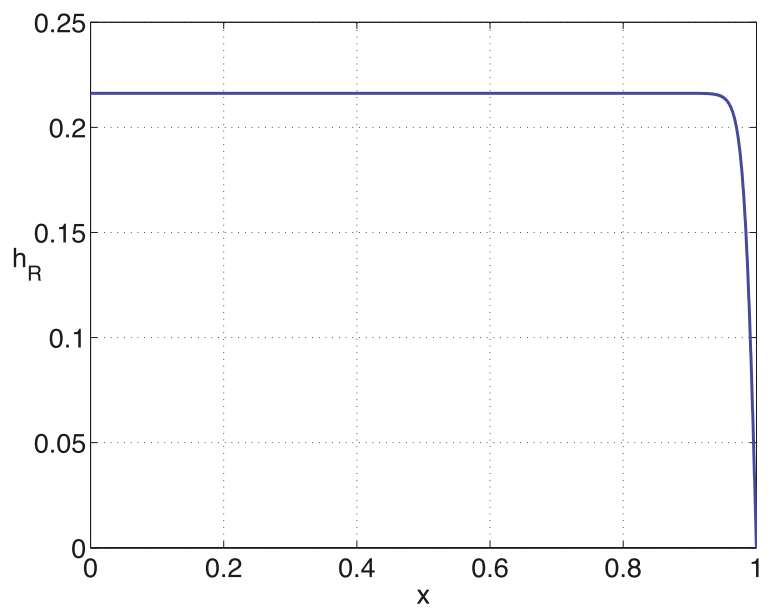

Figure 11. Plot of $h_{\mathcal{R}}(x)$.

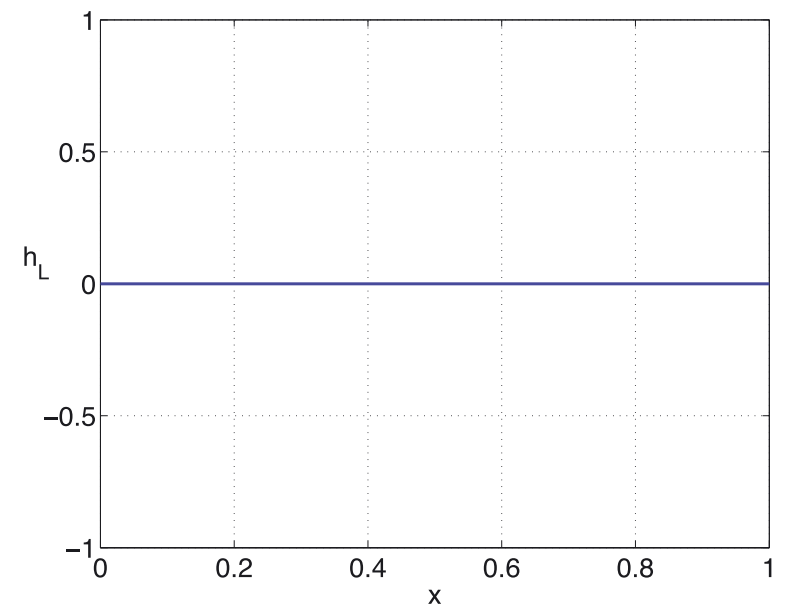

Figure 12. Plot of $h_{\mathcal{L}}(x)$.

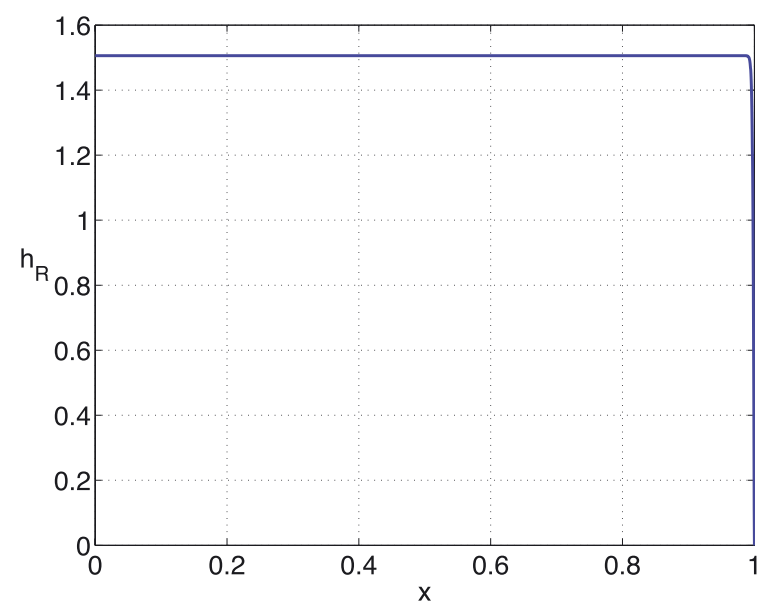

Figure 13. Plot of $h_{\mathcal{R}}(x)$. 
problem (5.17) - (5.20) for increasing times until the numerical solution has converged to the zero steady state. Since we have shown that the unique equilibrium $\hat{\varphi}(\cdot)=0$ is stable and all solutions converge uniformly to $\hat{\varphi}(\cdot)=0$, one would expect that the converged numerical solution would approximate the equilibrium solution $\hat{\varphi}(\cdot)=0$. As noted above, this does not always happen and in fact a converged numerical solution can be orders of magnitude away from $\hat{\varphi}(\cdot)=0$. We shall use the well known and convergent Crank-Nicolson scheme to explain why this happens.

The Crank-Nicolson scheme is a standard and generally stable finite-difference method for solving diffusion problems and similar partial differential equations. For Burgers' equations (5.17)-(5.20), the Crank-Nicolson scheme has the standard form:

$$
\begin{aligned}
\frac{z_{i, j+1}-z_{i, j}}{\Delta t}= & \frac{\nu}{2}\left[\frac{z_{i+1, j+1}-2 z_{i, j+1}+z_{i-1, j+1}}{h^{2}}+\frac{z_{i+i, j}-2 z_{i, j}+z_{i-1, j}}{h^{2}}\right] \\
& -\frac{1}{2}\left[z_{i, j+1} \frac{z_{i+1, j+1}-z_{i-1, j+1}}{2 h}+z_{i, j} \frac{z_{i+i, j}-z_{i-1, j}}{2 h}\right],
\end{aligned}
$$

with initial and boundary values

$$
\begin{aligned}
& z_{i, 0}=\phi\left(x_{i}\right) \text { for } i=1,2, \ldots, N \\
& z_{0, j}=z_{2, j} \text { for } j=0,1,2, \ldots, \\
& z_{N+1, j}=0 \text { for } j=0,1,2, \ldots
\end{aligned}
$$

Here, the space and time have been discretized, respectively, as $x_{i}=(i-1) h$ for $i=0,1,2, \ldots, N+1$ where $h=1 / N$, and $t_{j}=j \Delta t$ for $j=0,1, \ldots$ where the value $\Delta t$ is an increment in time $t$.

Observe that there are two (discrete) equilibrium solutions of the discrete system (5.23)-(5.27) denoted by $\boldsymbol{z}_{e, 1}$ and $\boldsymbol{z}_{e, 2}$. Specifically, $\boldsymbol{z}_{e, 1}=\mathbf{0}$ and

$$
\left(\boldsymbol{z}_{e, 2}\right)_{i}=\left\{\begin{array}{l}
\frac{2 \nu}{h} \text { for } \quad i=0,1,2, \ldots, N \\
0 \text { for } i=N+1
\end{array}\right.
$$

The first discrete equilibrium solution, $\boldsymbol{z}_{e, 1}$, exactly satisfies the Burgers' problem (5.17)-(5.20) at the discrete set of spatial points. However, the second discrete equilibrium solution, $\boldsymbol{z}_{e, 2}$, is not an approximation to the stationary Burgers' problem. For one thing, $\boldsymbol{z}_{e, 2}$ depends on the spatial discretization (i.e., $h$ or more precisely on $N$ ) so adjusting $h$ changes the solution. In addition, as $N$ increases the discrete equilibrium $\boldsymbol{z}_{e, 2}$ approaches infinity so it is not an approximation to a solution of the stationary Burgers' problem (5.21)-(5.22). Since, $\boldsymbol{z}_{e, 2}$ depends on the spatial discretization $(i . e ., h)$ as $N$ is increased, rather than obtaining a more accurate approximation of a fixed function, we obtain a totally different discrete solution.

On the other hand, for a fixed $N$, if the Crank-Nicolson method converges as $t$ increases (i.e., as $j \rightarrow \infty$ in (5.23)), then it must converge to an equilibrium solution at the nodal points. So, if the Crank-Nicolson method converges for this Burgers' problem as $j \rightarrow \infty$, then the numerical solution must converge to either $\boldsymbol{z}_{e, 1}$ or $\boldsymbol{z}_{e, 2}$. We note in addition, it was shown in [2] that the zero equilibrium solution, $\boldsymbol{z}_{e, 1}$, is stable. In a manner similar to the presentation in [2], it can also be shown that the non-zero equilibrium solution, $\boldsymbol{z}_{e, 2}$, is unstable.

Assume now that $\phi(\cdot)$ is smooth on $[0,1]$ so that $\phi\left(x_{i}\right) \neq\left(\boldsymbol{z}_{e, 2}\right)_{i}$ for $i=0,1 \ldots, N+1$. We have that if the Crank-Nicolson method converges for this Burger's problem, then necessarily $z_{i, j}$ must go to 0 as $j$ goes to infinity for $i=0,1,2, \ldots, N+1$. Depending on the initial starting values $z_{i, 0}$ we show that this is not the case. Namely, we first give an example to show that the Crank-Nicolson method converges and the numerical approximation converges to $\boldsymbol{z}_{e, 1}=\mathbf{0}$. Moreover, for a different set of starting values $z_{i, 0}$ the Crank-Nicolson 


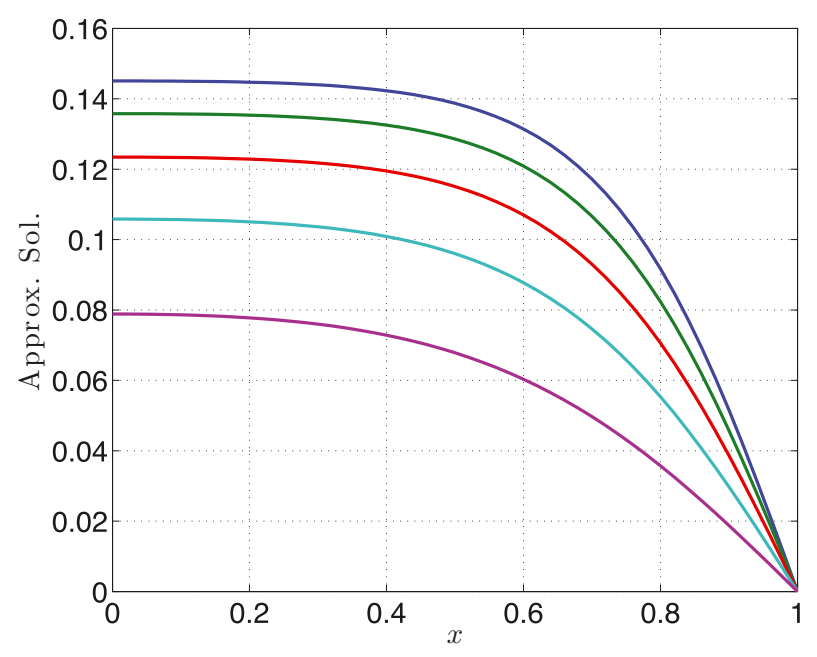

Figure 14. Approximate solutions to Burgers' problem at: $t=0$ (Blue), $t=80$ (Green), $t=120$ (Red), $t=160$ (Cyan), $t=200$ (Magenta).

method again converges to a discrete steady state, but the discrete function defined on the nodes is neither $\boldsymbol{z}_{e, 1}=\mathbf{0}$ nor $\boldsymbol{z}_{e, 2}$.

For this demonstration of what actually occurs for (5.23)-(5.27) we fix $\nu=1 / 50$ and choose an initial data $z_{i, 0}$ to be the values of a function $\phi(x)=M\left(1-x^{6}\right)$ at the nodes. We consider two different values of $M$. First we choose a small value $M=.152$ for which the result is exactly what is expected. The solution converges to $\boldsymbol{z}_{e, 1}=\mathbf{0}$ as $j \rightarrow \infty$. Let $N=20$ and set $\Delta t=0.25$. The computational solutions are displayed in Figure 14 for times of $0,80,120,160$ and 200 . As expected, the approximate solution decreases to the zero solution as the final time increases.

Now again consider $\nu=1 / 50$ but set $M=15.2$ and the initial distribution taken as $\phi(x)=15.2\left(1-x^{6}\right)$. Letting, for example, $N=500,750$ or 1000 and $\Delta t=0.00001,0.00005,0.0001$ or 0.0002 , the computational results are essentially identical. In these calculations, the computed solutions do not change after time greater than $t=0.01$. The computational solution has converged, i.e., the computations have converged in double precision arithmetic. Most importantly they are not converging to zero. The computed solutions are displayed in Figure 15 for times of $4,6,8$, and 10 , specifically when $N=500$ and $\Delta t=0.0001$. The computed solution is displayed in Figure 15 for times of 4, 6, 8, 10. and the value of the computed solution at $x=0$ produces either $h(0)=1.5200000$.

Given the computational results, it may be incorrectly inferred that the equilibrium solution to this problem is the one shown in Figure 15 as it is known that convergence is only obtained for this problem when the exact equilibrium solution is approached. The example thus illustrates that incorrect conclusions may possibly be made from computational results involving finite precision arithmetic.

The computational solution of this second problem is converging but to the equilibrium solution of the Burgers' problem (2.15) - (2.18) which is denoted by $h_{\mathcal{R}}(x)$ and defined in (3.12). Indeed, let us first recall that in double precision arithmetic $\alpha=2^{-1074}$ is the smallest positive nonzero number and $\alpha=2^{-1075}$ is set equal to zero. On the other hand $\sqrt{\alpha}=2^{-1075 / 2}$ is a nonzero number so we can solve the equation

$$
\sqrt{\frac{2}{\nu^{-1}}} R \operatorname{sech}(R)=F\left(R, \nu^{-1}\right)=\sqrt{\alpha}
$$




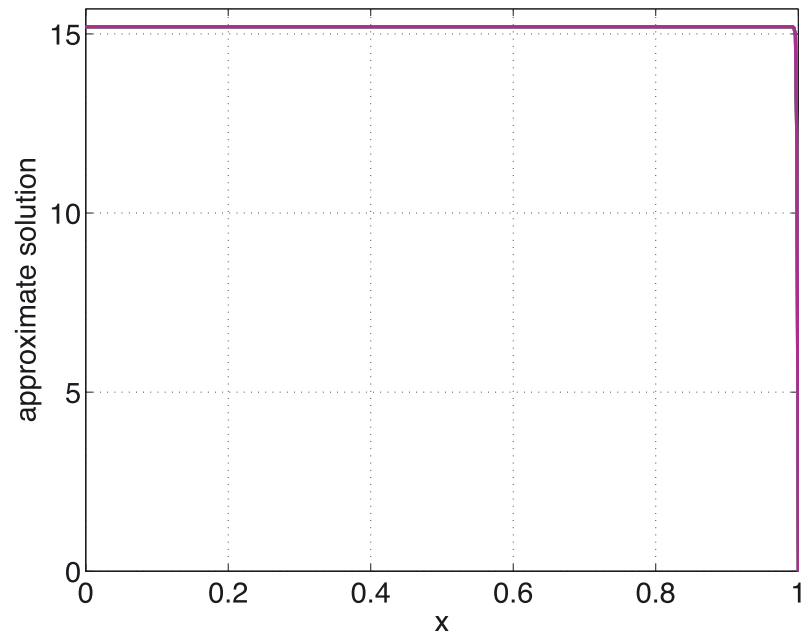

FIGURE 15. Converged calculated approximate solutions to Burgers' problem in double precision.

In this example we have set $\nu=1 / 50$ which gives $R_{\mathcal{R}}=377.584$ and

$$
h_{\mathcal{R}}(x)=\frac{2}{\nu^{-1}} R_{\mathcal{R}} \tanh \left(R_{\mathcal{R}}(1-x)\right)
$$

and hence

$$
h_{\mathcal{R}}(0)=15.1037 .
$$

Thus, by taking $M=15.2$ which is slightly larger than 15.1037 for the factor multiplying $\left(1-x^{6}\right)$, the numerical solution converges to a non-existent equilibrium for the homogeneous problem (5.17)-(5.20). However, this is an approximation of the nonhomogeneous problem $(2.15)-(2.18)$ with $\sqrt{\alpha}=2^{-1075 / 2}$. In summary, we have shown that;

\section{Remark 5.5.}

(i) The Crank-Nicolson scheme for $\alpha \neq 0$, is stable and converges as time increases to an equilibrium solution near the left solution $h_{\mathcal{L}}(x)$ for an initial condition near the left solution.

(ii) The Crank-Nicolson scheme for $\alpha=0$, has two discrete equilibrium solutions. The zero solution is stable and Crank-Nicolson scheme converges to the zero solution for an initial condition near zero. The nonzero equilibrium solution is unstable and depends on the spatial discretization level $h$. That is, if Crank-Nicolson converges as time increases and the discretization intervals decrease, it must converge to the zero solution.

(iii) The Crank-Nicolson scheme for $\alpha=0$ and a sufficiently large initial condition, converges computationally in finite precision arithmetic to a nonzero solution. Based on (5.5) above, this is not possible in infinite precision arithmetic.

What is important about (5.5) is that Crank-Nicolson scheme for $\alpha=0$ is converging in finite precision arithmetic to an incorrect solution. Indeed, we know that in exact arithmetic Crank-Nicolson cannot converge for this problem unless it is converging to the correct (zero) solution. The key difference is having to deal and compute with finite precision arithmetic.

\section{Concluding Remarks}

In this paper we have attempted to clarify an apparent numerical anomaly which the authors first observed many years ago for Burgers' equation with Neumann boundary conditions. We believe that this anomaly is important for the scientific computing community to keep in mind. This is especially true since the vast majority 
of scientific computing is performed using double precision floating point arithmetic. In addition, computational results are often not tested for errors due to finite precision arithmetic. It is precisely the floating point number system on a computer that allows this strange behavior to take place and produces erroneous solutions. It is an interesting side comment that this anomaly was discovered during investigations of control problems for conservation laws where the control enters through a Neumann boundary condition. Perhaps this is one reason this phenomenon was not previously observed in the computational science community since most numerical work on Burgers' equation focused on Dirichlet boundary conditions. Also, this is clearly a case where experimentation with computational mathematics (see [5]) has lead to a fundamental understanding of how computing on a finite precision machine can produce incorrect results even with theoretically convergent schemes. Finally, we note that we have investigated the stability properties of a Galerkin based numerical algorithm with similar results and conducted a rigorous sensitivity analysis of the problem (2.15)-(2.18) with respect to the parameter $\alpha$. The sensitivity analysis clearly shows that phenomenon discussed in this paper is due purely to computing in finite precision arithmetic and not "super-sensitivity". These results will appear in a future paper.

\section{ApPendix: Liouville transformation And REDUCTION to Sturm-Liouville Form}

To this end we let

$$
\eta=\exp \left(\int_{1}^{x} h(s) \mathrm{d} s\right)
$$

and consider a change of variables $\varphi=\eta \psi$. We need

$$
\begin{aligned}
\varphi_{x} & =\eta_{x} \psi+\eta \psi_{x} \\
\varphi_{x x} & =\eta_{x x} \psi+2 \eta_{x} \psi_{x}+\eta \psi_{x x} .
\end{aligned}
$$

Now we have

$$
\begin{aligned}
\eta & =2\left(\frac{1}{\nu^{-1}}\right) \operatorname{sech}(R(1-x)) \\
\eta_{x} & =2\left(\frac{1}{\nu^{-1}}\right) R \operatorname{sech}(R(1-x)) \tanh (R(1-x))=\frac{\nu^{-1} \eta h}{2} \\
\eta_{x x} & =\frac{\nu^{-1}\left(\eta_{x} \psi+\eta \psi_{x}\right)}{2} .
\end{aligned}
$$

Using these equations the equation is transformed into

$$
\left(\frac{1}{\nu^{-1}}\right) \eta \psi_{x x}-\frac{1}{2}\left[\eta_{x} h+h \eta-x\right] \psi=\lambda \eta \psi .
$$

Now to simplify this further we use the fact that

$$
\eta_{x}=\frac{\nu^{-1} \eta h}{2}
$$

to write

$$
\left(\frac{1}{\nu^{-1}}\right) \eta \psi_{x x}-\frac{1}{2}\left[\frac{\nu^{-1} h^{2}}{2}+h_{x}\right] \eta \psi=\lambda \eta \psi
$$

Since $\eta$ cannot be zero we can divide by it to obtain

$$
\left(\frac{1}{\nu^{-1}}\right) \psi_{x x}-\frac{1}{2}\left[\frac{\nu^{-1} h^{2}}{2}+h_{x}\right] \psi=\lambda \psi .
$$


We introduce another parameter $\beta$ by

$$
h^{\prime}(1)=-\beta .
$$

Now to simplify this further we use the fact that

$$
-\left(\frac{1}{\nu^{-1}}\right) h_{x}+\left(\frac{h^{2}}{2}\right)=c_{0}=\beta / \nu^{-1}
$$

Multiply both sides by $\nu^{-1}$ and rewrite to obtain

$$
\left(\frac{\nu^{-1} h^{2}}{2}\right)=h_{x}+\beta
$$

Plugging this into the formula above we obtain

$$
\begin{aligned}
\left(\frac{1}{\nu^{-1}}\right) \psi_{x x} & =\frac{1}{2}\left[\beta+2 h_{x}\right] \psi+\lambda \psi \\
& =\frac{1}{2}\left[\beta-2 \beta \operatorname{sech}^{2}\left(\sqrt{\left(\nu^{-1} \beta / 2\right)}(1-x)\right)\right] \psi+\lambda \psi \\
& =\frac{\beta}{2}\left[1-2 \operatorname{sech}^{2}\left(\sqrt{\left(\nu^{-1} \beta / 2\right)}(1-x)\right)\right] \psi+\lambda \psi .
\end{aligned}
$$

Next we recall that $R=\sqrt{\nu^{-1} \beta / 2}$ so that, multiplying both sides by $\nu^{-1}$, we finally arrive at

$$
\begin{aligned}
\psi_{x x}(x) & =\left(q(x)+\nu^{-1} \lambda\right) \psi(x), \quad x \in[0,1], \\
q(x) & =R^{2}\left[1-2 \operatorname{sech}^{2}(R(1-x))\right] .
\end{aligned}
$$

As for the boundary conditions we have

$$
0=\varphi(0)=(\eta \psi)_{x}(0)=\eta(0) \psi_{x}(0)+\eta(0) \psi_{x}(0)
$$

and $\eta_{x}=\nu^{-1} \eta h / 2$ and $\nu^{-1} h(0) / 2=R \tanh (R)$ to get

$$
\psi_{x}(0)+R \tanh (R) \psi(0)=0 .
$$

And at $x=1$ we have

$$
0=\varphi(1)=\eta(1) \psi(1) \quad \Rightarrow \quad \psi(1)=0 .
$$

Combining these results we arrive at the desired regular Sturm-Liouville problem

$$
\begin{aligned}
\psi_{x x}(x) & =\left(q(x)+\nu^{-1} \lambda\right) \psi(x), \quad x \in[0,1], \\
q(x) & =R^{2}\left[1-2 \operatorname{sech}^{2}(R(1-x))\right] \\
\psi_{x}(0) & +k(R) \psi(0)=0, \quad k(R)=R \tanh (R) \\
\psi(1) & =0 .
\end{aligned}
$$




\section{REFERENCES}

[1] V.S. Afraimovich, M.K. Muezzinoglu and M.I. Rabinovich, Metastability and Transients in Brain Dynamics: Problems and Rigorous Results, in Long-range Interactions, Stochasticity and Fractional Dynamics; Nonlinear Physical Science, edited by Albert C.J. Luo and Valentin Afraimovich. Springer-Verlag (2010) 133-175.

[2] E. Allen, J.A. Burns, D.S. Gilliam, J. Hill and V.I. Shubov, The impact of finite precision arithmetic and sensitivity on the numerical solution of partial differential equations. Math. Comput. Model. 35 (2002) 1165-1195.

[3] E. Allen, J.A. Burns and D.S. Gilliam, On the use of numerical methods for analysis and control of nonlinear convective systems, in Proc. of 47th IEEE Conference on Decision and Control (2008) 197-202.

[4] J.A. Atwell and B.B. King, Stabilized Finite Element Methods and Feedback Control for Burgers' Equation, in Proc. of the 2000 American Control Conference (2000) 2745-2749.

[5] D.H. Bailey and J.M. Borwein, Exploratory Experimentation and Computation, Notices AMS 58 (2011) 1410-1419.

[6] A. Balogh, D.S. Gilliam and V.I. Shubov, Stationary solutions for a boundary controlled Burgers' equation. Math. Comput. Model. 33 (2001) 21-37.

[7] M. Beck and C.E. Wayne, Using Global Invariant Manifolds to Understand Metastability in the Burgers Equation With Small Viscosity. SIAM Review 53 (2011) 129-153 [Published originally SIAM J. Appl. Dyn. Syst. 8 (2009) 1043-1065].

[8] T.R. Bewley, P. Moin and R. Temam, Control of Turbulent Flows, in Systems Modelling and Optimization, Chapman and Hall CRC, Boca Raton, FL (1999) 3-11.

[9] J.T. Borggaard and J.A. Burns, A PDE Sensitivity Equation Method for Optimal Aerodynamic Design. J. Comput. Phys. 136 (1997) 366-384.

[10] J. Burns, A. Balogh, D. Gilliam and V. Shubov, Numerical stationary solutions for a viscous Burgers' equation. J. Math. Syst. Estim. Control 8 (1998) 1-16.

[11] J.A. Burns and S. Kang, A control problem for Burgers' equation with bounded input/output. Nonlinear Dyn. 2 (1991) 235-262.

[12] J.A. Burns and S. Kang, A Stabilization problem for Burgers' equation with unbounded control and observation, in Estimation and Control of Distributed Parameter Systems. Int. Ser. Numer. Math. vol. 100, edited by W. Desch, F. Rappel, K. Kunisch. Springer-Verlag (1991) 51-72.

[13] J.A. Burns and H. Marrekchi, Optimal fixed-finite-dimensional compensator for Burgers' Equation with unbounded input/output operators. ICASE Report No. 93-19. Institute for Comput. Appl. Sci. Engrg., Hampton, VA. (1993).

[14] J.A. Burns and J.R. Singler, On the Long Time Behavior of Approximating Dynamical Systems, in Distributed Parameter Control, edited by F. Kappel, K. Kunisch and W. Schappacher. Springer-Verlag (2001) 73-86.

[15] C.I. Byrnes and D.S. Gilliam, Boundary control and stabilization for a viscous Burgers' equation. Computation and Control, Progress in Systems Control Theory, vol. 15. Birkhäuser Boston, Boston, MA (1993) 105-120.

[16] C.I. Byrnes, D.S. Gilliam and V.I. Shubov, Convergence of trajectories for a controlled viscous Burgers' equation, Control and Estimation of Distributed Parameter Systems: Nonlinear Phenomena. Int. Ser. Numer. Math., vol. 118, edited by W. Desch, F. Rappel, K. Kunisch. Birkhäuser, Basel (1994) 61-77.

[17] C.I. Byrnes, D. Gilliam, V. Shubov and Z. Xu, Steady state response to Burgers' equation with varying viscosity, in Progress in Systems and Control: Computation and Control IV, edited by K. L.Bowers and J. Lund. Birkhäuser, Basel (1995) 75-98.

[18] C.I. Byrnes, D.S. Gilliam and V.I. Shubov, High gain limits of trajectories and attractors for a boundary controlled viscous Burgers' equation. J. Math. Syst. Estim. Control 6 (1996) 40.

[19] C.I. Byrnes, A. Balogh, D.S. Gilliam and V.I. Shubov, Numerical stationary solutions for a viscous Burgers' equation. J. Math. Syst. Estim. Control 8 (1998) 16 (electronic).

[20] C.I. Byrnes, D.S. Gilliam and V.I. Shubov, On the Global Dynamics of a Controlled Viscous Burgers' Equation. J. Dyn. Control Syst. 4 (1998) 457-519.

[21] C.I. Byrnes, D.S. Gilliam and V.I. Shubov, Boundary Control, Stabilization and Zero-Pole Dynamics for a Nonlinear Distributed Parameter System. Int. J. Robust Nonlinear Control 9 (1999) 737-768.

[22] C. Cao and E. Titi, Asymptotic Behavior of Viscous Burgers' Equations with Neumann Boundary Conditions, Third Palestinian Mathematics Conference, Bethlehem University, West Bank. Mathematics and Mathematics Education, edited by S. Elaydi, E. S. Titi, M. Saleh, S. K. Jain and R. Abu Saris. World Scientific (2002) 1-19.

[23] M.H. Carpenter, J. Nordström and D. Gottlieb, Revisiting and extending interface penalties for multi-domain summation-byparts operators. J. Sci. Comput. 45 (2010) 118-150.

[24] J. Carr and J.L. Pego, Metastable patterns in solutions of $u_{t}=\epsilon^{2} u_{x x}-f(u)$. Comm. Pure Appl. Math. 42 (1989) 523-576.

[25] J. Carr, D.B. Duncan and C.H. Walshaw, Numerical approximation of a metastable system. IMA J. Numer. Anal. 15 (1995) 505-521.

[26] C.A.J. Fletcher, Burgers' equation: A model for all reasons, in Numerical Solutions of J. Partial Differ. Eqns., edited by J. Noye. North-Holland Publ. Co. Amsterdam (1982) 139-225.

[27] A.V. Fursikov and R. Rannacher, Optimal Neumann Control for the 2D Steady-State Navier-Stokes equations, in New Directions in Math. Fluid Mech. The Alexander. V. Kazhikhov Memorial Volume. Advances in Mathematical Fluid Mechanics, Birkhauser, Berlin (2009) 193-222.

[28] G. Fusco, G. and J. K. Hale, Slow-motion manifolds, dormant instability, and singular perturbations. J. Dyn. Differ. Eqns. 1 (1989) 75-94.

[29] T. Gallay and C.E. Wayne, Invariant manifolds and the long-time asymptotics of the navier-stokes and vorticity equations on $\mathbb{R}^{2}$. Arch. Rational Mech. Anal. 163 (2002) 209-258. 
[30] T. Gallay and C.E. Wayne, Global stability of vortex solutions of the two-dimensional Navier-Stokes equation. Commun. Math. Phys. 255 (2005) 97-129.

[31] M. Garbey and H.G. Kaper, Asymptotic-Numerical Study of Supersensitivity for Generalized Burgers' Equation. SIAM J. Sci. Comput. 22 (2000) 368-385.

[32] S. Gottlieb, D. Gottlieb and C.-W. Shu, Recovering High-Order Accuracy in WENO Computations of Steady-State Hyperbolic Systems. J. Sci. Comput. 28 (2006) 307-318.

[33] M. Gunzburger, L. Hou and T. Svobodny, Analysis and finite element approximation of optimal control problems for the stationary Navier-Stokes equations with distributed and Neumann controls. Math. Comput. 57 (1991) 123-151.

[34] M.D. Gunzburger, H.C. Lee and J. Lee, Error estimates of stochastic optimal Neumann boundary control problems. SIAM J. Numer. Anal. 49 (2011) 1532-1552.

[35] J.S. Hesthaven, S. Gottlieb and D. Gottlieb, Spectral Methods for Time Dependent Problems, Cambridge Monographs on Applied and Computational Mathematics, vol. 21. Cambridge University Press (2006).

[36] IEEE Computer Society, IEEE Standard for Binary Floating-Point Arithmetic, IEEE Std 754-1985 (1985).

[37] A. Kanevsky, M.H. Carpenter, D. Gottlieb and J. S. Hesthaven, Application of implicit-explicit high order Runge-Kutta methods to discontinuous-Galerkin schemes. J. Comput. Phys. 225 (2007) 1753-1781.

[38] R. Kannan and Z.J. Wang, A high order spectral volume solution to the Burgers' equation using the Hopf-Cole transformation. Int. J. Numer. Meth. Fluids (2011). Available on wileyonlinelibrary.com. DOI: 10.1002/fld.2612.

[39] O.A. Ladyzhenskaya, V.A. Solonnikov and N.N. Ural'ceva, Linear and Quasilinear Equations of Parabolic Type, Translations of the AMS, vol. 23 (1968).

[40] J.G.L. Laforgue and R.E. O'Malley, Supersensitive Boundary Value Problems, Asymptotic and Numerical Methods for Partial Differential Equations with Critical Parameters, edited by H.G. Kaper and M. Garbey. Kluwer Publishers (1993) 215-224.

[41] H.V. Ly, K.D. Mease and E.S. Titi, Distributed and boundary control of the viscous Burgers' equation. Numer. Funct. Anal. Optim. 18 (1997) 143-188.

[42] H. Marrekchi, Dynamic Compensators for a Nonlinear Conservation Law, Ph.D. Thesis, Department of Mathematics, Virginia Polytechnic Institute and State University, Blacksburg, VA, 24061 (1993).

[43] V.Q. Nguyen, A Numerical Study of Burgers' Equation With Robin Boundary Conditions, M.S. Thesis. Department of Mathematics, Polytechnic Institute and State University, Blacksburg, VA, 24061 (2001).

[44] P. Pettersson, J. Nordström and G. Laccarino, Boundary procedures for the time-dependent Burgers' equation under uncertainty. Acta Math. Sci. 30 (2010) 539-550.

[45] J.T. Pinto, Slow motion manifolds far from the attractor in multistable reaction-diffusion equations. J. Differ. Eqns. 174 (2001) 101-132.

[46] S.M. Pugh, Finite element approximations of Burgers' Equation, M.S. Thesis. Departmant of Mathematics, Polytechnic Institute and State University, Blacksburg, VA, 24061 (1995).

[47] G.R. Sell and Y. You, Dynamics of Evolutionary Equations, vol. 143. Springer-Verlag (2002).

[48] Z.-H. Teng, Exact boundary conditions for the initial value problem of convex conservation laws. J. Comput. Phys. 229 (2010) $3792-3801$.

[49] M.J. Ward and L.G. Reyna, Internal layers, small eigenvalues, and the sensitivity of metastable motion. SIAM J. Appl. Math. 55 (1995) 425-445.

[50] T.I. Zelenjak, Stabilization of solutions of boundary value problems for a second-order parabolic equation with one space variable, Differentsial'nye Uravneniya 4 (1968) 34D45.

[51] T.I. Zelenyak, M.M. Lavrentiev Jr. and M.P. Vishnevskii, Qualitative Theory of Parabolic Equations, Part 1, VSP, Utrecht, The Netherlands (1997). 